\title{
Hierarchical structure in language and action: A formal comparison
}

\author{
Cas W. Coopmans ${ }^{1,2 *}$, Karthikeya Kaushik ${ }^{1,3}$, \& Andrea E. Martin ${ }^{1,3}$ \\ ${ }^{1}$ Max Planck Institute for Psycholinguistics, Nijmegen, The Netherlands \\ ${ }^{2}$ Centre for Language Studies, Radboud University, Nijmegen, The Netherlands \\ ${ }^{3}$ Donders Institute for Brain, Cognition and Behaviour, Radboud University, Nijmegen, The Netherlands
}

\begin{abstract}
Since the cognitive revolution, language and action have been compared as cognitive systems, with crossdomain convergent views recently gaining renewed interest in biology, neuroscience, and cognitive science. Language and action are both combinatorial systems whose mode of combination has been argued to be hierarchical, combining elements into constituents of increasingly larger size. This structural similarity has led to the suggestion that they rely on shared cognitive and neural resources. In this paper, we compare the conceptual and formal properties of hierarchy in language and action using set theory. We show that the strong compositionality of language requires a particular formalism, a magma, to describe the algebraic structure corresponding to the set of hierarchical structures underlying sentences. When this formalism is applied to actions, it appears to be both too strong and too weak. To overcome these limitations, which are related to the weak compositionality and sequential nature of action structures, we formalize the algebraic structure corresponding to the set of actions as a trace monoid. We aim to capture the different system properties of language and action in terms of the distinction between hierarchical sets and hierarchical sequences, and discuss the implications for the way both systems could be represented in the brain.
\end{abstract}

Keywords: Hierarchy, syntax, compositionality, formal modeling, set theory

\section{*Contact details:}

Cas W. Coopmans

Max Planck Institute for Psycholinguistics

Wundtlaan 1, 6525 XD Nijmegen, The Netherlands

Tel.: +31-24 3521331

Email address: cas.coopmans@mpi.nl 


\section{Introduction}

It has long been recognized that both language and action are structurally organized in a way that is not immediately evident from their serial appearance. In the 1950s, Lashley (1951) and Chomsky (1959) separately showed that then dominant behaviorist "chaining" theories based on contiguous stimulus-response associations could not account for 'serial behavior', such as language production and action execution. Instead, these behaviors appear to be controlled by internal, hierarchically organized plans, which allow human behavior to be creative, productive and flexible. Since then, similarities between language and action have often been noted (e.g., Corballis, 1991; Greenfield, 1991; Holloway, 1969; Miller et al., 1960), and more recent studies propose that language and action are analogous in their hierarchical organization (Fitch \& Martins, 2014; Fujita, 2014; Jackendoff, 2007; Pulvermüller \& Fadiga, 2010; Stout \& Chaminade, 2009).

Such proposals about cross-domain convergence are desirable from an evolutionary perspective, in which one seeks to find a set of primitives that account for the distinguishing features of the human mind (Boeckx \& Fujita, 2014; de Waal \& Ferrari, 2010; Hauser et al., 2002; Marcus, 2006). However, arguments in favor of the analogy between language and action are formally underspecified. It is possible to draw a hierarchical tree structure over any sequence, but what is needed is independent empirical evidence that this structure describes or explains a phenomenon in the natural world (Berwick \& Chomsky, 2017; Bloom, 1994; Fitch \& Martins, 2014; Moro, 2014a). In other words, superficial resemblance is insufficient: "we cannot just observe that hierarchical structures are found in motor control (e.g., tool construction), and thereby claim that these are directly related to the hierarchical structures of language ... Rather, it is necessary to develop a functional description of the cognitive structures in question, parallel to that for language ... so we can look for finer-scale commonalities" (Jackendoff, 2002, p. 80).

While formal linguistics has provided many accounts of the specific properties of hierarchy in language, such a formal characterization in the domain of actions and action plans is lacking (but see Steedman, 2002 for an exception). To this end, the aim of this paper is to characterize the similarities and differences between the hierarchical structures in language and action in both conceptual and formal terms (cf. Guest \& Martin, 2021; van Rooij \& Blokpoel, 2020). The paper is structured as follows: in Section 2, we discuss the type of data that shows that the syntax of natural languages is organized hierarchically, after which we list the core properties of such hierarchical syntactic structure (Section 2.1). In Section 2.2, we formally describe these structures in a domain-neutral way using the mathematical language of set theory. We then show that this formalism is inadequate for describing the action system (Section 3.1) and suggest an alternative formalism to characterize its properties (Section 3.2). In Section 4, we conclude that the properties of syntactic hierarchy are not found in action structures (Section 4.2) and discuss this conclusion in light of the idea that syntactic representations are fundamentally hierarchical sets, while actions are better conceived of as hierarchical sequences (Section 4.3). We end by discussing the implications for how language and action might be represented in the brain.

\section{Hierarchical structure in language}

In linguistics, the term hierarchy refers to the format of linguistic representations. At all levels of organization (phrases, words and syllables), linguistic structure is organized hierarchically (see Everaert et al., 2015 for a recent overview). In the domain of syntax specifically, it refers to the fact that words are embedded into constituents, which are in turn recursively embedded into larger constituents, creating the hierarchically organized syntactic structures that are often visually denoted by means of tree structures. These tree structures are graphic representations of relations which are essentially set-theoretic (Lasnik, 2000).

A main source of evidence for constituency is the observation that the interpretation of phrases and sentences is often determined by structural relationships. For example, the sentence "the woman saw the man with binoculars" has two meanings. Either the woman has binoculars, which she uses to look at the man, or the man has binoculars. The sentence is ambiguous because it corresponds to two possible structures, which differ in terms of the attachment site of the prepositional phrase (PP) "with binoculars" (see (1)). If it attaches to "the man", forming a complex noun phrase (NP) constituent (1a), the man has the binoculars, but if it attaches to the verb phrase (VP) "saw the man" (1b), the woman must be holding the binoculars. Here, it is the structural relationship between the PP and the other constituents that determines how the sentence is interpreted. 
The structure dependence of meaning shows that language is compositional. To be able to compare combinatorial systems, such as language and action, we distinguish between strong and weak compositionality (Pagin \& Westerståhl, 2010). In a strongly compositional system, the meaning of a constructed unit is a function of the meanings of its constituents and the way in which these are structurally combined (Partee et al., 1993; Partee, 1995). In a weakly compositional system, instead, the meaning of a constructed unit is a function of the meaning of the elements and the total construction (i.e., the result of an operation applied over the total construction of ordered elements; Pagin \& Westerståhl, 2010). A weakly compositional system can thus distinguish the meanings of "John likes Mary" and "Mary likes John", because their total constructions differ. However, weakly compositional systems cannot capture structural ambiguity. Because they do not take into account the structural relationships between intermediate representations, such as between the different constituents in (1), they are unable to distinguish the two interpretations of "the woman saw the man with binoculars".

(1) a.

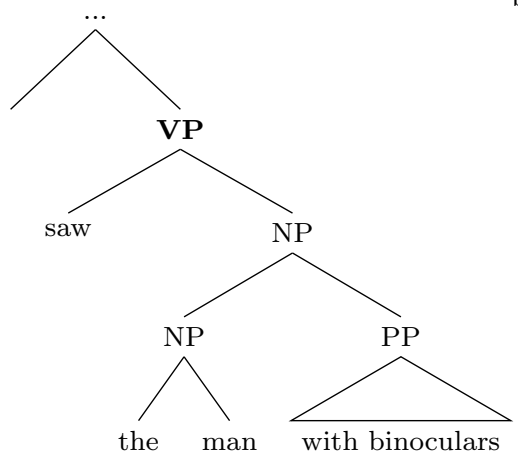

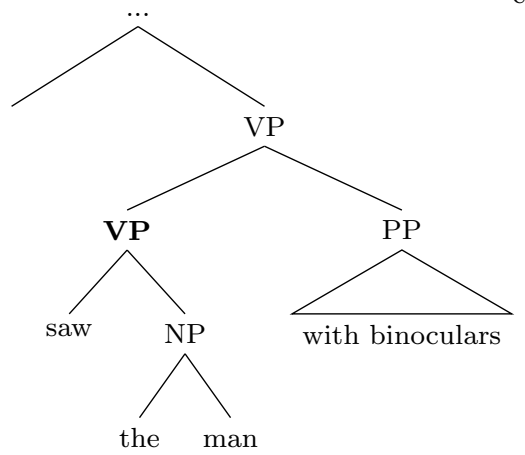

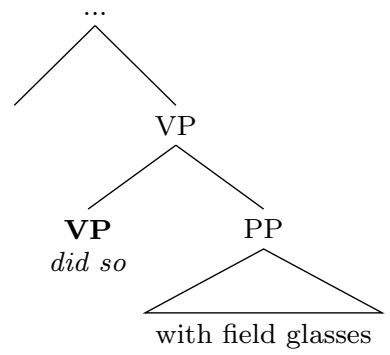

A second source of evidence for constituent structure is that syntactic operations, such as deletion and substitution, target constituents rather than words or mere word sequences. For instance, the phrase did so can substitute for a verbal word sequence, such as "saw the man", if this sequence forms a constituent. Because the words "saw the man" form an isolated constituent only in the structure of (1b), the sentence "the woman saw the man with binoculars and the boy did so with field glasses" (corresponding to (1c)) can only mean that the boy is holding the field glasses (analogous to the interpretation of (1b)), not the man. In sum, both semantic interpretation and syntactic operations are structure-dependent: they refer to hierarchical constituent structures rather than to linear sequences of words, with the result that word sequences that do not form constituents are not available to semantic interpretation nor to syntactic operations.

\subsection{Properties of syntactic structure}

To generate such hierarchical structure, (any theory of) the language faculty must include, at a minimum, a computational procedure for combining smaller elements into larger elements. The properties of this procedure are debated, but all linguistic frameworks assume it in one form or another: Merge in the Minimalist Program (Chomsky, 1995b), Unify in the Parallel Architecture (Jackendoff, 2002), Forward/Backward Application in Combinatory Categorial Grammar (Steedman, 2000), and Substitution in Tree-Adjoining Grammar (Joshi \& Schabes, 1997). For our purposes, we do not need to select among these frameworks; all we assume is that the generation of syntactic structures relies on a combinatorial operation, whose properties must be formally defined, and which must be computationally general enough so that it could play a role in cognitive domains beyond language (Fukui \& Zushi, 2004; Rizzi, 2004). Merge is one of the operators that meets these requirements, as it is formally defined as binary set formation: $\operatorname{Merge}(\alpha, \beta)$ takes two elements $\alpha$ and $\beta$ and forms the unordered set $\{\alpha, \beta\}$ (Chomsky, 2013; Collins, 2017). It can be applied recursively, such that is takes its own output as input: further combining $\gamma$ with the already formed set $\{\alpha, \beta\}$ yields the set $\{\gamma,\{\alpha, \beta\}\}$. As should be clear, recursive application of this combinatorial operation yields a structure that is hierarchical: the smaller set is contained in the larger set. Because the generated set is unordered (i.e., $\{\gamma,\{\alpha, \beta\}\}$ is identical to $\{\{\beta, \alpha\}, \gamma\}$ ), the elements in the set cannot be described in terms of linear precedence. Rather, the relevant relationships are established with respect to structure: the element $\gamma$, which 
is "higher" in the structure, has a structurally more prominent position than the elements $\alpha$ and $\beta$.

In the remainder of this paper, we will assume that the combinatorial procedure for generating structure is formally equivalent to binary set formation. On this assumption, the hierarchical structure of syntax has the following properties: ${ }^{1}$

1. Unbounded. Human language use is creative: language users can produce and understand sentences that have never been produced before. Specifying such an open-ended capacity using finite means requires recursive procedures, such as the recursive combinatorial operation defined above. Given the controversy surrounding (the importance of) recursion and the various uses of the term in different disciplines (Fitch, 2010; Martins, 2012; Watumull et al., 2014), we should first be explicit about the relationship between recursion and hierarchy.

While the hierarchical structure of syntax is generated by recursion, hierarchy and recursion are two independent properties. Hierarchy is a property of the output generated by the combinatorial operation (i.e., a property of its extension). Recursion, instead, is a property of a function defined in intension. A recursive function is a function which can apply indefinitely to its own output ('self-reference'), which leads to structurally 'self-similar' output, in which a unit of a specific type is contained in another unit of the same type (in linguistics, this is often called 'self-embedding': embedding of one thing into another thing of the same kind). This results in a hierarchical structure which displays similar properties across different levels of embedding, clearly visible in the repetition of complement clauses like He said that she believes that he thought..., which is a sentence within a sentence within a sentence. Because a recursive function is defined in intension rather than in extension, and thus the (infinite) computable set is not the same as the (finite) set actually produced by the function, the recursivity of a function should not be equated with its output. Therefore, absence of 'self-similar' output does not warrant the conclusion that the function which generated the output is not recursive (Watumull et al., 2014; Hauser et al., 2014), and neither does the presence of 'self-similar' output warrant the definitive conclusion that it was generated via recursion (Martins, 2012).

The independence of hierarchy and recursion is further illustrated by the fact that they doubly dissociate: not all hierarchical objects are generated by recursion and not all recursive functions generate hierarchical structure. For instance, artificial grammars that generate sequences of the type (ab) ${ }^{n}$ and $\mathrm{a}^{n} \mathrm{~b}^{n}$ can be recursive (e.g., via respectively $f: \mathrm{S} \rightarrow$ abS and $f: \mathrm{S} \rightarrow \mathrm{aSb}$ ), but only the latter generates hierarchical structure ${ }^{2}$. Conversely, the syllable structure in phonology is hierarchical but not recursive. A syllable contains an onset and a rhyme, with the latter consisting of a nucleus and a coda. This hierarchy is not recursive: a syllable cannot be embedded in another syllable.

2. Endocentric. The categorial status of a constituent is determined by one of its elements (the 'head'): the set $\{\alpha, \beta\}$ can be of type $\alpha$ or $\beta$, but not of type $\gamma$. Endocentric structures are contrasted with exocentric structures, in which the label of a composed unit is not determined by one of its elements ${ }^{3}$. Labels allow phrases to be called upon by interpretive and formal procedures, thereby determining their distributional behavior. To give an example, the set \{eat, cookies\} is a verb phrase, which has 'eat-like' (interpretative) semantic properties and 'verb-like' (formal) syntactic properties, both inherited from the verb "eat". That this is the case can be seen from the fact that "eat cookies" can take the place of the verb "eat" in "He likes to eat", yielding "He likes to eat cookies". It cannot, however, take the place of the noun "cookies" in "He likes chocolate cookies", as is clear from the ill-formedness of "He likes chocolate eat cookies". The label of a composed unit thus places a constraint on further computation,

\footnotetext{
${ }^{1}$ More properties of language can be derived from the minimal assumption that the structure-building procedure is binary set formation (see Hornstein, 2017 and Rizzi, 2013 for comprehensive lists of properties). However, many of these properties, such as displacement, do not have clear analogues in actions (Moro, 2015; Pulvermüller, 2014). Because our aim is to compare the (formal) properties that language and actions might share, we focus on the properties of hierarchy listed here.

${ }^{2}$ The grammars that generate $(\mathrm{ab})^{n}$ and $\mathrm{a}^{n} \mathrm{~b}^{n}$ sequences can be implemented recursively, though they do not have be. These sequences can also be generated with iterative functions that are not recursive, i.e., do not call themselves (Fitch, 2010; Jackendoff, 2011). Iterative functions also realize unboundedness, but they do so by creating sequences without internal structure.

${ }^{3}$ How it is determined which element defines the label of the phrase is still a much-debated question and is outside the scope of this paper (see e.g., Boeckx, 2009; Chomsky, 2013; Fukui, 2011). What is important here is not how phrases get their labels, but that they get them from one of their elements. Moreover, by using the term 'labels' we only refer to the fact that the combined unit is of the same type as one of its elements. Whether these labels reflect phrasal projections from the syntactic category of a lexical item (as in X-bar theory, Jackendoff, 1977) or rather the lexical item itself (as in bare phrase structure, Chomsky, 1995a) is not critical for out purposes.
} 
restricting the elements with which it can combine: given that \{eat, cookies\} is a verb phrase and not a noun phrase, it can combine with adverbs but not with adjectives.

Endocentricity is intricately linked to recursivity, because the combinatorial operation can only be said to apply recursively if its output is of the same type as its input (Boeckx, 2009; Hornstein, 2009; Watumull et al., 2014). Similar to recursivity, endocentricity is a distinctive property of syntactic hierarchy, as not all linguistic structures are endocentric.

3. Unordered. Because the combinatorial operation is defined as binary set formation, no order is imposed on the members in the combined set. While the unordered structure has to be linearized for spoken language production, differences in linear order do not feed differences in semantic interpretation, and syntactic operations do not refer to linear order. Different languages (and different modalities) can seem highly different in terms of the linear ordering of their words (e.g., whether heads precede or follow their dependents), which is a fundamental source of cross-linguistic variation (see Section 4). However, in terms of the compositional structure generated by Merge, which is what we are concerned with, these languages show consistent similarities. Note that the assumption about unordereness is specific to the definition of Merge as binary set formation, and might not be shared in other linguistic frameworks ${ }^{4}$. What these frameworks do agree on, however, is that syntactic operations are structure-dependent, not order-dependent.

This conception of structure building as binary set formation allows us to derive both compositionality and structure dependence. First, the structure of the input to the combinatorial operation is preserved in its output. Thus, if $\alpha$ and $\beta$ are constituents (or sets) in the input, they are constituents (or sets) in the output as well: new elements can only be added on top of the already formed set, not inside it. Because the structure of every combination is retained at each level of the hierarchy, the hierarchical structure is strongly compositional. This is well illustrated with a structurally ambiguous phrase: $\{$ deep, $\{$ blue, sea $\}$ \} is not the same as $\{\{$ deep, blue $\}$, sea $\}$. Note that if the structure were not retained after recursive combination, it would be possible to derive from $\{$ blue, sea $\}$ not only $\{$ deep, $\{$ blue, sea $\}\}$ but also $\{\{$ deep, blue $\}$, sea $\}$. That would make it impossible to account for the ambiguity of the phrase.

Moreover, recursively generated sets describe hierarchical relations but not linear ordering relations. Therefore, syntactic operations that refer to these sets can only refer to its structure, and hence be structuredependent, but not to its order. Order-dependent operations are also ruled out by recursion: because it is always possible to recursively insert material between two items and thereby change the linear position of the words (e.g., "the boy swims" $\rightarrow$ "the boy with muscular arms swims"), no operation can refer to the linear position of elements in a sequence.

We should note that the properties we described above are properties of a cognitive capacity, which can be expressed in varying degrees in natural languages (e.g., exocentricity might be found in certain subjectpredicate relations). Moreover, the faculty of language is capable of assigning strongly compositional interpretations to most sentences, as is required to derive the multiple interpretations of structurally ambiguous sentences, but it can assign other interpretations as well (e.g., to non-decomposable idioms; Baggio, 2021; Jackendoff, 2002). In other words, we listed properties that a model of (the faculty of) language must have, even though these need not be found in all constructions in all languages. As we aim to illustrate how the action system differs from the language system, we will focus on the capacity for strong compositionality as a fundamental difference between both systems.

\subsection{Formalizing linguistic structure}

In order to be able to evaluate the similarities and differences between the hierarchical structure of language and action in a transparent way, we need a theory-neutral conceptual vocabulary to describe these structures. Ideally, this description should be accompanied by a formal analysis of the similarities and differences, as well as an evaluation of their implications (Guest \& Martin, 2021; Martin, 2016, 2020; O'Donnell et al., 2005; Partee et al., 1993; van Rooij \& Blokpoel, 2020). To this end, the following paragraphs will present a formal model in which we incorporate the properties of syntactic structure as defined in Section 2.1.

\footnotetext{
${ }^{4}$ See Saito and Fukui (1998) and Kayne (2011), who argue that Merge $(\alpha, \beta)$ forms the ordered pair $\langle\alpha, \beta\rangle$. This makes immediate precedence part of syntax.
} 


\subsubsection{Generating structures}

Definition 1. $(M, \oplus, \varnothing)$ is a unital, commutative magma generated from a set $W$, where

1. $W$ is the set of words that represents the lexicon of a language.

2. $M$ is a set of elements that are generated from $W$, with $W \subset M$.

3. $\oplus$ is a binary set formation operation, such that for $\forall a, b \in M, a \oplus b=\{a, b\}=b \oplus a \in M$. Additionally, $\oplus$ is non-associative, so $\forall a, b, c \in M,(a \oplus b) \oplus c \neq a \oplus(b \oplus c)$.

4. $\varnothing$ is the identity element, such that $\forall m \in M, m \oplus \varnothing=m=\varnothing \oplus m$.

A unital, commutative magma (henceforth referred to as a magma for conciseness) is an algebraic structure (see Box 1), whose operation we define as binary set formation following the formal definition of Merge described in Section 2.1. This allows us to derive a number of important properties. First, as the magma axiom states that for any two members $a, b \in M$, application of this operator to $a$ and $b$ generates a member of $M$, thus yielding unbounded generation. Second, $\oplus$ does not introduce labels, so the label of each set is derived from one of its elements (i.e., endocentricity; see Chomsky, 2013; Collins, 2017) ${ }^{5}$. Third, all elements in $M$ are unordered sets. And fourth, $\oplus$ is non-associative, which means that the order in which $\oplus$ is applied affects the structure that is generated (Fukui \& Zushi, 2004). In other words, the structures that are generated are strongly compositional: their meaning is a function of the meanings of their parts and the way in which they are structurally combined.

\section{Box 1. Algebraic structures}

An algebraic structure consists of a nonempty set $X$ (called the carrier set), a collection of finitary operations on $X$ (typically binary operations), and a finite set of axioms that these operations must satisfy. To illustrate the relevant axioms for the current work, we consider $X$ as the set containing all elements of the algebraic structure and $\odot$ as a binary operation acting on the elements of $X$.

(1.) $\forall x_{1}, x_{2} \in X, x_{1} \odot x_{2} \in X$

closed

(2.) $\forall x \in X, \exists ! i \in X$ such that $x \odot i=i \odot x=x$ unital

(3.) $\forall x_{1}, x_{2}, x_{3} \in X,\left(x_{1} \odot x_{2}\right) \odot x_{3}=x_{1} \odot\left(x_{2} \odot x_{3}\right)$ associative

(4.) $\forall x_{1}, x_{2} \in X, x_{1} \odot x_{2}=x_{2} \odot x_{1}$

commutative

Depending upon axioms they satisfy, the algebraic structures form a taxonomy. Presented below is a subset of this taxonomy, in which we highlight both the algebraic structures that are relevant for the current work as well as their corresponding axioms.

\section{Unital}

Commutative

Magma

Closed
Magma

Unital

Commutative

\section{Monoid}

Unital

Associative

\section{Commutative}

Monoid

Unital

Associative

Commutative

\section{Trace}

Monoid

Unital

Associative

Partially

commutative

Without further constraints, a freely generated magma would contain elements that should not be constituents, such as $\{\{e a t\},\{h a p p y\}\}$. To avoid this without modifying the formal properties of $\oplus$, the lexical

\footnotetext{
${ }^{5}$ Labels are a convenient way to group together structures with identical formal properties. In our formal setup, constituent labels are simply part labels whose union produces the set of all grammatical structures. For example, with $W=\{d o g, m a n, b i g\}$, the label $N$ would be the part $\{$ man, $\operatorname{dog}\}, A$ is $\{b i g\}$, and $N P$ is $\{\{b i g, \operatorname{dog}\},\{b i g, \operatorname{man}\}\}$. Therefore, $M=M_{N} \bigcup M_{A} \bigcup M_{N P}$.
} 
items themselves must determine which structures are licensed and which are not. That is, the application of $\oplus$ is constrained by selectional restrictions on its input (i.e., which categories can(not) combine with which other categories). For instance, $\{\{e a t\},\{h a p p y\}\}$ is excluded because verbs do not combine with adjectives. The same restrictions apply when the output of $\oplus$ is recursively used as its input. For example, the set $\{\mathrm{v}\{$ eat $\},\{$ cookies $\}\}$ cannot combine with the adjective "happy" because the former is labeled as a type of verb rather than as a type of noun. Such illegitimate combinations are excluded by taking the (grammatically licensed) subset of the freely generated magma.

We make the relationship between these constituent structures explicit by defining a binary relationship between the elements of the magma, turning it into a partially ordered magma.

Definition 2. $(M, \oplus, \varnothing, \leq)$ is a partially ordered magma, where $\leq i s$ a containment relationship between the elements in $M$ that is reflexive, transitive, and antisymmetric.

The relation $\leq$ on the set $M$ reflects containment or set-inclusion, which corresponds to the dominance relation commonly used in linguistics. Thus, $x_{1} \leq x_{2}$ means that $x_{2}$ contains (and thus dominates) $x_{1}$. As a visualization of this partially ordered magma, consider the Hasse diagram in Figure 1, which displays the containment relationship for two structures that map onto the sequence "woman saw man with binoculars".

\section{Box 2. Ordered sets}

An ordered set $X$ is a set ordered by a binary relation, denoted here with infix notation $\leq$, such that $\forall x, y, z \in X$, the following axioms may hold (depending on the kind of order):

(1.) $x \leq x$; reflexive

(2.) if $x \leq y$ and $y \leq z$, then $x \leq z$; transitive

(3.) if $x \leq y$ and $y \leq x$, then $x=y$; antisymmetric

(4.) $x \leq y$ or $y \leq x$

total

When the binary relation is transitive and antisymmetric, the set is called partially ordered. A totally ordered set is an ordered set whose binary relation holds between all elements of the set. When a relationship is only total when restricted to $X^{\prime}$, which is a subset of $X$, we consider $X^{\prime}$ locally total (Kayne, 1994). We therefore say that $\forall x, y \in X^{\prime} \subset X, x \leq y$ or $y \leq x$.

Besides containment, there is another relevant structural relation between the elements in constituent structures. This relationship, called c-command (Reinhart, 1983), describes the scope domain of a node in the tree structure. Specifically, a node $\alpha$ is said to asymmetrically c-command a node $\beta$ iff $\beta$ is contained in the sister node of $\alpha$ (e.g., in (1a) "saw" c-commands every node contained in the higher NP).

Definition 3. For $m_{1}, m_{2} \in M, m_{1}$ c-commands $m_{2}$ (denoted $m_{1} \gg m_{2}$ ) if $m_{1} \not \leq m_{2}$, and $m_{2} \not \leq m_{1}$, and $\exists ! m=\left\{m_{1}, x\right\} \in M$, and $m_{2} \leq x$. An asymmetric c-command relationship exists between $m_{1}$ and $m_{2}$ if $m_{1} \gg m_{2}$ and $m_{2} \gg m_{1}$. Asymmetric c-command is irreflexive, transitive, antisymmetric, and locally total.

Given Definition 3, asymmetric c-command is a locally total relation on non-terminal nodes in the tree structure $^{6}$. The Hasse diagram in Figure 2 visualizes the asymmetric c-command relationship for the two structures that map onto the sequence "woman saw man with binoculars".

\subsubsection{Sequences}

Definition 4. $(S, *, ")$ is a monoid generated by $W$, where

1. $W$ is the set of words that represents the lexicon of a language.

2. $S$ are the sequences generated from $W$, with $W \in S$.

\footnotetext{
${ }^{6}$ Strictly speaking, the relation is left-locally total (Kayne, 1994). A left-locally total relation is total only on the elements to the left of the relation (e.g., for $a R b, R$ is left-locally total for $a$ ).
} 


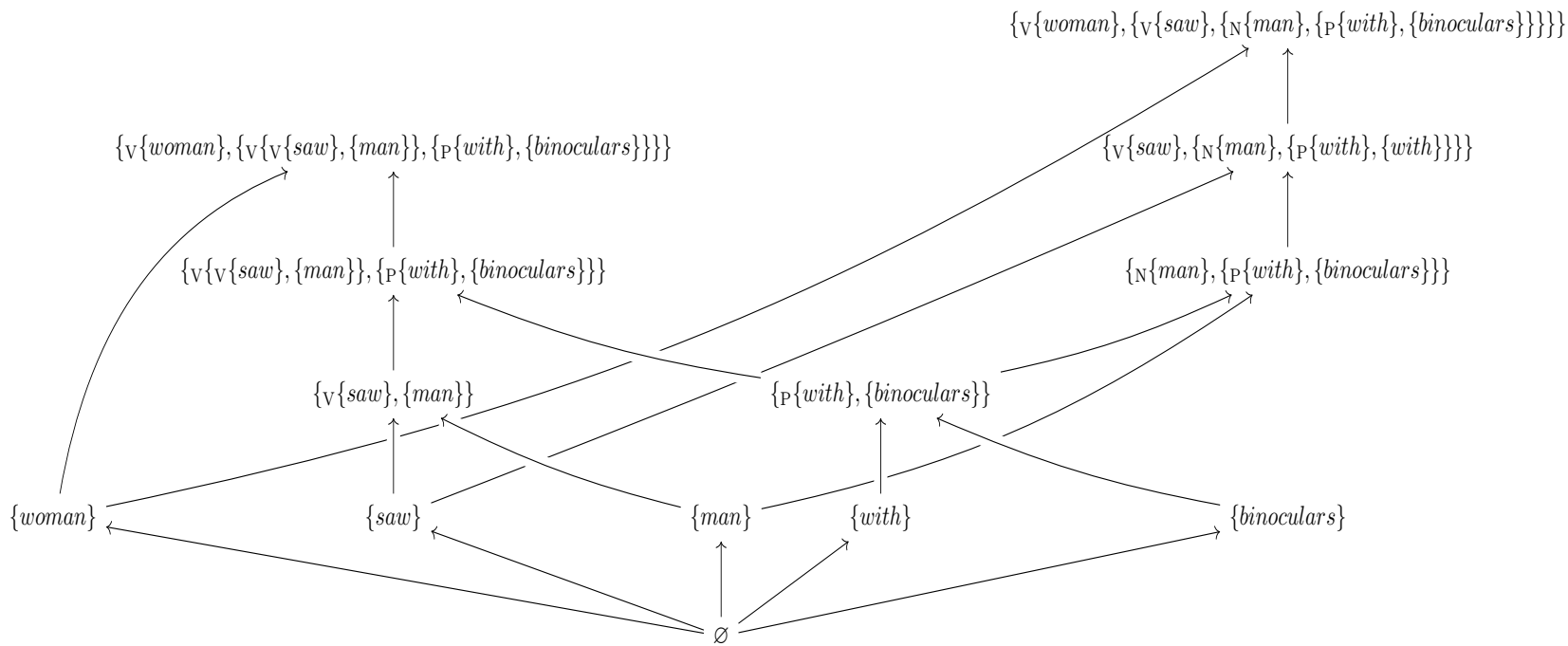

Figure 1: Hasse diagram of a subset of the partially ordered magma $M$, which displays two different structures that map onto the sequence "woman saw man with binoculars". Arrows indicate direct containment. The subscript at the opening curly bracket of each binary set indicates the label of that set.

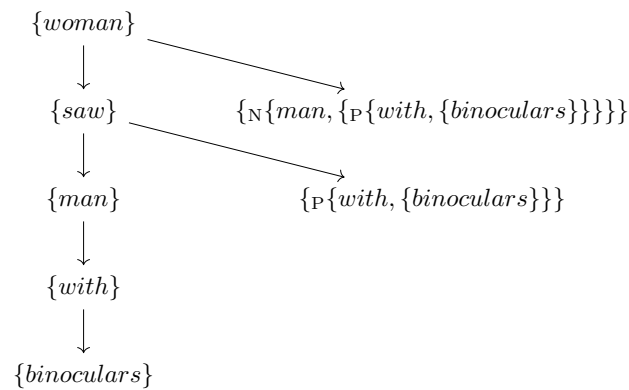

(a.)

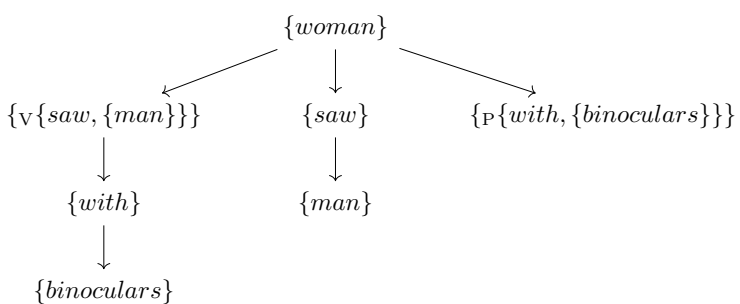

(b.)

Figure 2: Hasse diagram of a subset of the partially ordered magma $M$, which displays two different structures that map onto the sequence "woman saw man with binoculars". Arrows indicate asymmetric c-command. The subscript at the opening curly bracket of each binary set indicates the label of that set. The diagram in (a) corresponds to the structure in (1a), where the man has the binoculars. The diagram in (b) corresponds to the structure in $(1 \mathrm{~b})$, in which the woman is holding the binoculars. 
3. * is the concatenation operation, which is unital and associative.

4. The empty sequence " is the identity element.

Definition 5. We define a binary relation $(\prec)$ on the sub-sequences in $s=\left(x_{1}, x_{2}, \ldots x_{n}\right) \in S$, which we call precedence, where $x_{1} \prec x_{2} \prec \ldots x_{n}$. Precedence is irreflexive, transitive, antisymmetric, and locally total.

Given Definition 5, precedence is a locally total relation on the set of elements in a sequence (i.e., the set of terminal nodes in the tree structure). The Hasse diagram in Figure 3 visualizes the precedence relationship for the sequence "woman saw man with binoculars".

woman $\longrightarrow$ saw $\longrightarrow$ man $\longrightarrow$ with $\longrightarrow$ binoculars

Figure 3: Hasse diagram of an element of the set of sequences $S$, which displays the sequential structure of the sequence "woman saw man with binoculars". Arrows indicate precedence.

\subsubsection{Mapping structures to sequences}

Following Kayne (1994), we assume that there exists a rigid mapping between hierarchical structure and linear order, such that only one linear sequence can be derived from a given hierarchical structure. As noted above, asymmetric c-command and precedence are locally total orders on the set of non-terminals and the set of terminals, respectively. Kayne (1994) formalizes the mapping between these two orders in the Linear Correspondence Axiom.

\section{Linear Correspondence Axiom (LCA):}

A lexical item $\alpha$ precedes a lexical item $\beta$ iff

(i) $\alpha$ asymmetrically c-commands $\beta$ or

(ii) an XP dominating $\alpha$ asymmetrically c-commands $\beta$

Definition 6. We adopt the LCA as a surjective function $f: M \rightarrow S$, defining f for a pair of lexical items $\alpha, \beta \in m \in M$, which holds for all elements of the sequence by induction:

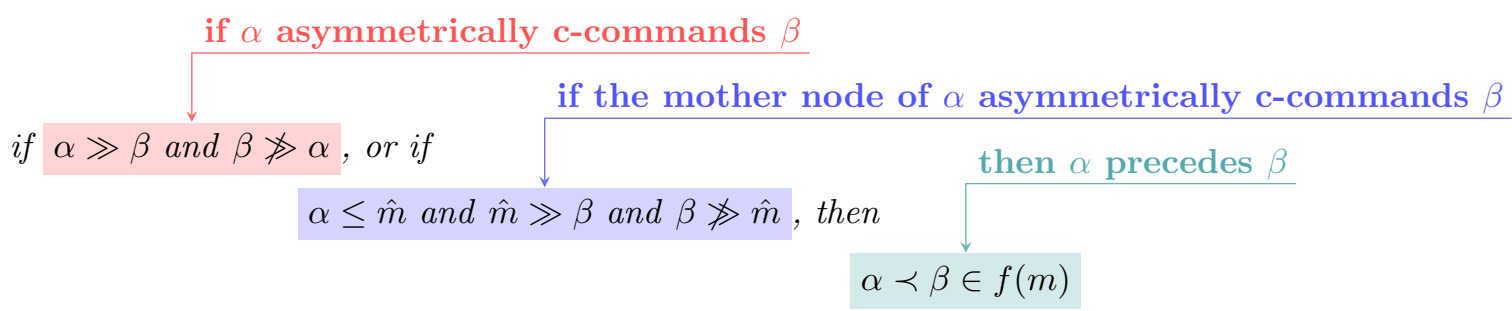

In short, Definition 6 states that a word $\alpha$ precedes a word $\beta$ if it asymmetrically c-commands $\beta$ or if the projection of $\alpha$ asymmetrically c-commands $\beta$. The result of this mapping is a full total ordering of the terminals of the hierarchical structure in question. It is important to note that this mapping can be defined as a proper function because, under the LCA, only one linear sequence can be derived from any given hierarchical structure. Conversely, multiple hierarchical structures can map onto the same linear sequence. For instance, the precedence relations that are derived from the asymmetric c-command relations in the two structures in Figure 2 are the same, which illustrates the fact that the corresponding sequence is structurally ambiguous.

\subsubsection{Ordering sequences via structures}

When the sets in $M$ are mapped to sequences in $S$, these sequences are imbued with grammatical properties. What these grammatical properties are can be understood in terms of the ordering that is carried over from 
the containment relation in $M$. Consider Figure 4, where the constituent structures in $M$ (left panel) are mapped to the sequences in $S$ (middle panel). By virtue of the containment relation by which the elements of $M$ are ordered, this mapping imposes a structure on the set of sequences (right panel) that is not there if only their sequential properties are considered.

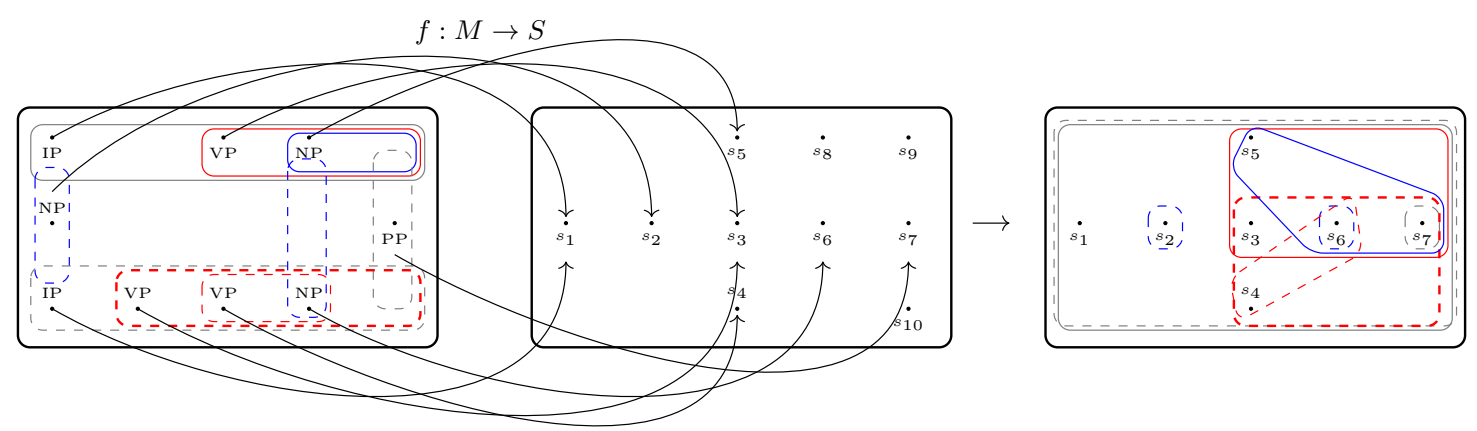

$$
\begin{array}{ll}
s_{1} \text { : woman saw man with binoculars } & s_{6}: \text { man } \\
s_{2}: \text { woman } & s_{7}: \text { with binoculars } \\
s_{3}: \text { saw man with binoculars } & s_{8}: \text { man with } \\
s_{4}: \text { saw man } & s_{9}: \text { saw man with } \\
s_{5}: \text { man with binoculars } & s_{10}: \text { man man with with }
\end{array}
$$

Figure 4: An ordering relationship is imposed on $S$ via the structure in $M$. The leftmost panel contains a subset of the partially ordered magma $M$, with the elements (denoted by their labels) ordered by containment. The labels IP, VP, NP and PP refer to the labels of the constituents. Here, IP stands for Inflectional Phrase (whose head contains information about tense and inflection), which is often used as the top-most constituent in the structural representation of a sentence. A subset of $S$ is shown in the middle panel, with example sequences presented below the figure. The LCA function $f: M \rightarrow S$ maps elements in $M$ to elements in $S$, thus imposing a structural ordering relation on the sequential elements in $S$ (rightmost panel).

If we only consider the sequential properties of the elements in $S$, a partial ordering already exists. This partial ordering is based on string containment. For example, both "woman with" and "with binoculars" can be said to be contained in the sequence "woman with binoculars". Using the map $f: M \rightarrow S$, we impose a restriction on this ordering: for two elements $m_{1}, m_{2} \in M, f\left(m_{1}\right) \leq f\left(m_{2}\right)$ if $m_{1} \leq m_{2}$. That is, two sequences in $S$ are contained in one another only if their constituent structures in $M$ are contained in one another. This imposed ordering restricts the initial ordering by excluding both ungrammatical sequences as well as containment relations that are not the result of a structural relationship. For example, in the middle panel of Figure 4, the subsequences $s_{8}, s_{9}$, and $s_{10}$ do not appear in the imposed partial ordering. $s_{10}$ is an ungrammatical sequence and therefore has no valid structural analog in $M . s_{8}$ and $s_{9}$ are subsequences of a grammatical sequence, yet they do not correspond to constituents and are therefore not retained in the ordering. Thus, only strings that correspond to constituents are retained in the partial ordering, and this partial ordering is based on constituent containment, as can be seen in the substructure in the right panel of Figure 4.

To sum up, we used the binary set formation operator $\oplus$ to generate hierarchical constituent structure. From the resulting structure, whose containment relationships are visualized in Figure 1, we derive all ccommand relationships (see Figure 2). From these c-command relationships we derive a linear sequence with precedence relationships using the LCA. Using the containment relationship in the partially ordered magma (see Figure 1), we impose an ordering relation on the resulting set of sequences (see Figure 4). The latter is possible because we define the algebraic structure corresponding to the set of structures as a magma, whose combinatorial operator is non-associative. This allows us to generate strongly compositional structure, which is a necessary requirement for any description of (the faculty of) language. 


\section{Hierarchical structure in actions}

Having defined and formalized the properties of hierarchical structure in syntax, we will now consider whether action hierarchies are analogous to syntactic hierarchies. Similar to the hierarchical structure underlying sentences, action sequences are thought to be governed by hierarchically organized action plans (Botvinick, 2008; Cooper \& Shallice, 2000, 2006; Holloway, 1969; Koechlin \& Jubault, 2006; Lashley, 1951; Miller et al., 1960; Rosenbaum et al., 2007) ${ }^{7}$. This structural analogy between linguistic syntax and actions has recently received considerable attention from several corners of cognitive science (Boeckx \& Fujita, 2014; Fadiga et al., 2009; Jackendoff, 2007, 2009; Moro, 2014a, 2014b; Stout \& Chaminade, 2009), in which the hierarchical structure of actions is thought to be generated by an action syntax (Fitch \& Martins, 2014; Fujita, 2014; Maffongelli et al., 2019; Pulvermüller, 2014).

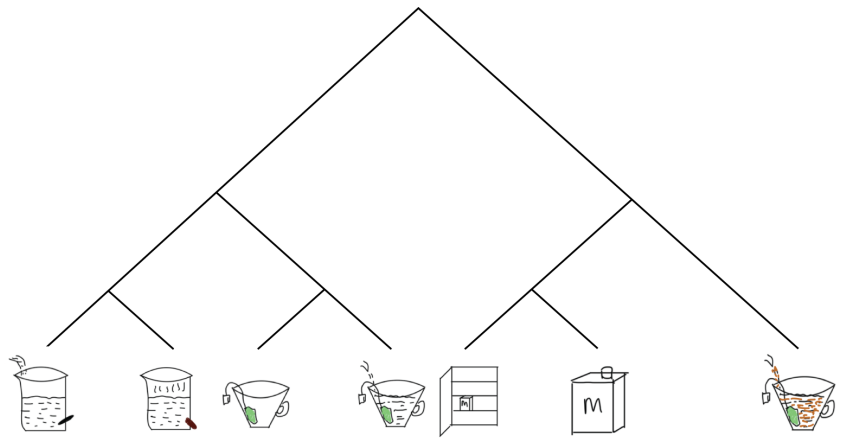

Figure 5: An example of a hierarchical decomposition of an action sequence, such as making tea. The terminal nodes correspond to the atomic actions in Figure 6.

The idea is often illustrated using the example of tea- or coffee-making as a goal-directed behavioral routine (Cooper \& Shallice, 2000; Fitch \& Martins, 2014; Fischmeister et al., 2017; Humphreys \& Forde, 1998; Jackendoff, 2007, 2009; Kuperberg, 2020). A multi-step action, such as tea-making, can be decomposed into discrete subsequences of actions, which in turn can be decomposed in sub-subsequences, and so on. Figure 5 shows a visual representation of the hierarchical part-whole structure of 'making tea'. The highest level in the hierarchy represents the complex, temporally extended and goal-directed action, middle levels represent short-term, less complex subactions with their own subgoals, and the lowest level (terminal nodes) contains atomic actions with immediate subgoals. Decomposing complex actions into these embedded (sub)sequences is theoretically and empirically warranted because the subsequences may be used in different tasks, because they are sometimes unintentionally omitted, repeated, or substituted as a whole, and because they all have their own (sub)goal, which must be fulfilled in order to achieve the overarching goal (Cooper \& Shallice, 2000, 2006; Humphreys \& Forde, 1998; Lashley, 1951; Norman, 1981; Reason, 1979; Rosenbaum et al., 2007; Schwartz, 2006).

\subsection{Formalizing action structure (1)}

The following sections describe the structure of actions using the same mathematical formalism used to describe language in Section 2.2. We first show that this formalism is inadequate for describing actions. Section 3.2 proposes an alternative way to describe the structure of action sequences.

Definition 7. $(M, \oplus, \varnothing)$ is a unital, commutative magma generated from a set A, where

1. A is a set containing atomic actions, such as the example presented in Figure 6.

2. $M$ is a set of elements that are generated from $A$, with $A \subset M$.

\footnotetext{
${ }^{7}$ Note that we are concerned with describing the structure of actions rather than with describing how action sequences come about in a processing system (Badre, 2008; Tettamanti \& Moro, 2012). The latter question belongs to the study of motor control, which is also hierarchically organized but which has different properties: motor control is based on causal relations ('processing' hierarchy), while actions should be described in terms of part-whole relations ('representational' hierarchy; see Uithol et al., 2012 for discussion).
} 
3. $\oplus$ is a binary set formation operation, which is non-associative and closed.

4. $\varnothing$ is the identity element.

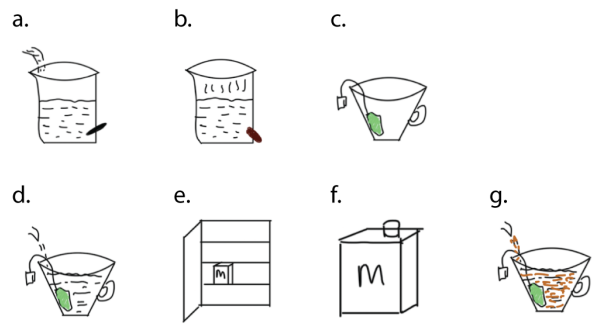

Figure 6: Atomic actions for tea making. (a) fill kettle with water. (b) turn on kettle. (c) put teabag in cup. (d) pour hot water into cup. (e) open fridge. (f) grab milk. (g) pour milk into cup. In the context of Definition $7, A=\{a, b, c, d, e, f, g\}$.

By defining the same binary relationship as used in Definition 2, we derive a partially ordered magma in which the actions and action sets are partially ordered by containment. A subset of this partially ordered magma is visualized in the Hasse diagram in Figure 7, which displays the containment relationship for two structures that map onto the same action sequence for making tea. Note that the two top-most action structures are derived in a different way. This figure illustrates a crucial point about the (ir)relevance of hierarchical structure in the interpretation of action sequences. That is, because the $\oplus$ operator is non-associative, the order in which actions are combined using $\oplus$ affects the structure that is generated. Therefore, if we were to interpret these structures in a strongly compositional way, we would have to conclude that they correspond to different actions. This is clearly an undesirable conclusion, because the two structures correspond to one and the same action sequence. In other words, adopting a non-associative combinatorial operator for generating action structures makes the model too strong: it will differentiate two action structures that should not be distinguished because they map onto the same action sequence and thus achieve the same goal in effectively the same way.

\subsubsection{Compositionality in language and action}

The fact that a strongly compositional formal model does not accurately describe actions indicates that the action system is not strongly compositional. If the action system is weakly compositional instead, it follows that one action sequence cannot be associated with multiple hierarchical structures. This prediction is borne out: structurally ambiguous actions, where one action sequence is associated with more than one structural representation and therefore more than one goal, do not seem to exist. This does not mean that actions cannot be ambiguous. Any given action may be characterized in terms of different goals, but these different 'goals' are not a function of a decomposition of the action sequence in terms of hierarchically organized 'action constituents'. Whether the action's goal is achieved depends on the temporal order of its constituent actions, not on their hierarchical organization.

\subsection{Formalizing action structure (2)}

In the previous section we showed that when the language formalism is applied to actions, it appears to be too strong: it makes a distinction it should not be making. The model is also too weak: actions and action plans are structured by temporal (precedence) relations, but the model does not take temporal order into account. In the current section, we therefore propose an alternative way to describe the structure of actions. The operator(s) used to generate action structures must meet at least two requirements. First, it must generate sequential structure, because actions are temporally ordered. Second, it must not be non-associative, because actions are not strongly compositional. 


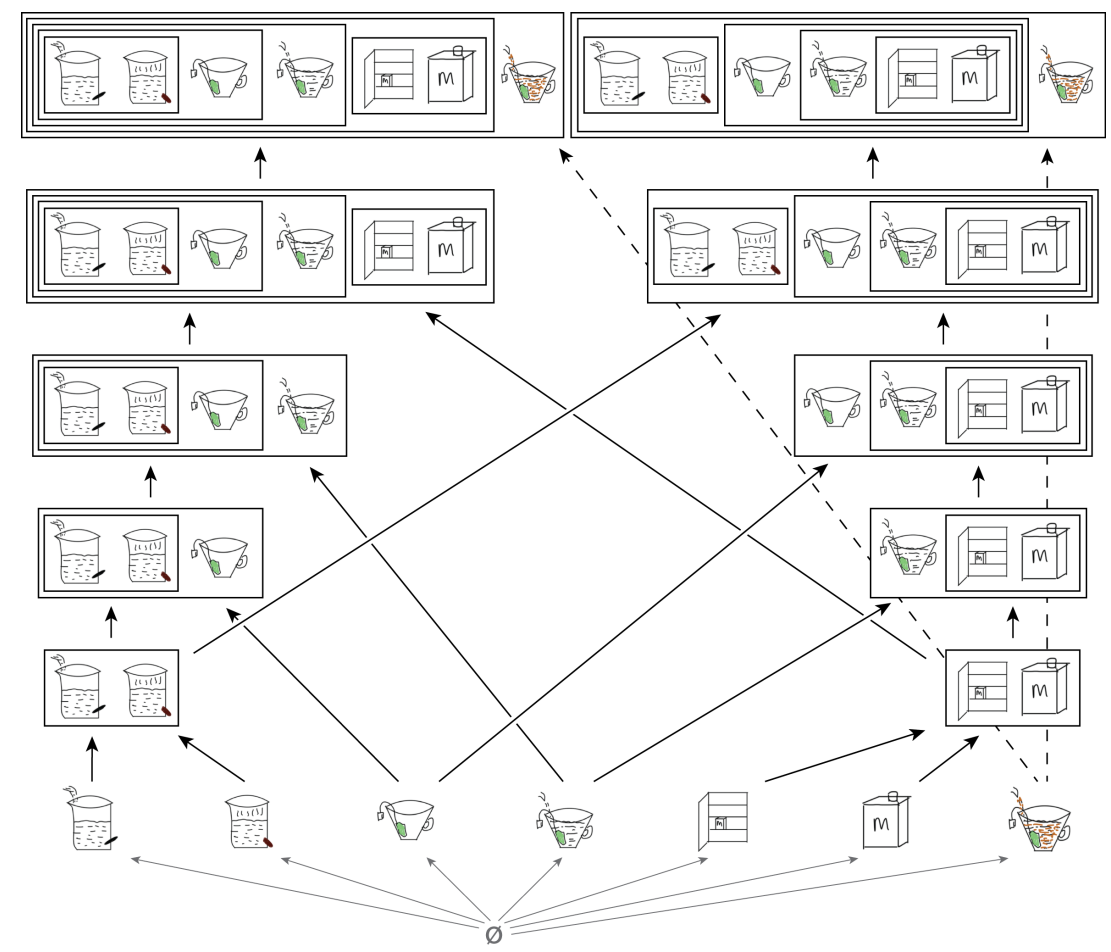

Figure 7: Hasse diagram of a partially ordered magma, which displays two different structures that map onto the exact same action sequence for making tea. The boxes around action combinations represent binary sets, and the arrows indicate direct containment.

\subsubsection{A set of partitioned sequences}

We have already defined the set of sequences as $S$ (see Definition 4). The elements in each sequence are in a total, transitive, and antisymmetric ordering (see Box 2). This set is partitioned according to the following criterion: sequences are deemed equivalent if they bring about a particular change in the environment (i.e., they achieve the same 'goal'). All equivalent sequences are part of a single equivalence class, whose label corresponds to the goal achieved by the sequences in it.

Definition 8. Given the set $S$, a partition of $S$ contains a set $G$, and for each $g \in G$, a non-empty subset $S_{g} \subseteq S$ exists, such that:

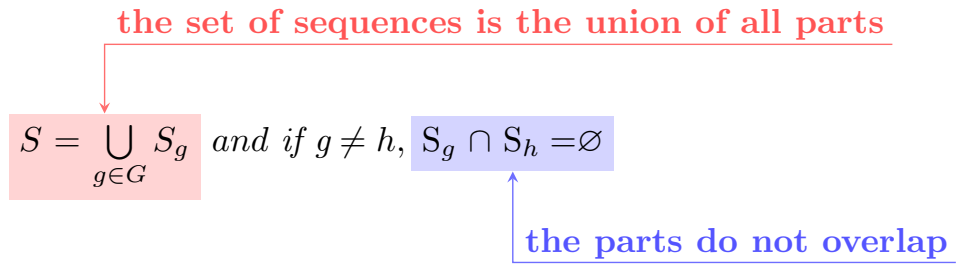

Here, we take $g \in G$ as the set of part labels (i.e., the labels or 'names' given to each element in the partition). Because all sequences in a given part are equivalent, we call every element $s \in S_{g}$ a representative sequence of that part $S_{g}$.

The partitioning of $S$ yields a set of part labels that correspond to the set of goals they accomplish. These goals can be interpreted as abstractions over action sequences that have something in common, namely the change they bring about in the environment (see e.g., Cooper \& Shallice, 2000). 


\subsubsection{Generating structured sequences}

Definition 9. We define action structure as $(G, \otimes, *, \varnothing)$, where:

1. The elements of $G$ are part labels (see Definition 8) corresponding to action sequences that generate a particular goal.

2. $\otimes$ and $*$ are two sequence building operators that generate the elements of $G$, with $\varnothing$ as the identity element.

Note that we include the set of atomic actions in $G$ since it can be argued that an atomic action has the same status of a goal in that it can be decomposed into smaller sub-actions, each achieving a particular change in the environment. Therefore, an atomic action is simply an equivalence class with only one element.

A goal can often be achieved in several ways. For example, given the actions in Figure 6, the goal 'make black tea' corresponds to the part $S_{b}$, where $S_{b}=\{(a, b, c, d),(a, c, b, d),(c, a, b, d)\}$. Here, $a$ ('fill kettle with water') must precede $b$ ('turn on kettle'), which in turn must precede $d$ ('pour hot water into cup'), so the temporal ordering of $(a, b, d)$ is fixed. However, the position of action $c$ ('put teabag into cup') within this action sequence is specified only in relation to $d$; it can be placed at any position before $d$ within $(a, b, d)$, thus yielding three action sequences. In other words, for a given goal to be achieved, the temporal ordering of some actions must be specified, whereas it need not be specified for other actions. We achieve this combination of the requirement of strict temporal ordering with temporal flexibility via the use of two sequence building operators.

Definition 10. $*$ is a sequence building operation $*: G \times G \rightarrow G$. Let $a, b \in G$ be two part labels, and $s_{a} \in S_{a}$ and $s_{b} \in S_{b}$ be two representative sequences, where $s_{a}=\left(a_{1}, a_{2}, \ldots a_{n}\right), s_{b}=\left(b_{1}, b_{2}, \ldots b_{m}\right)$.

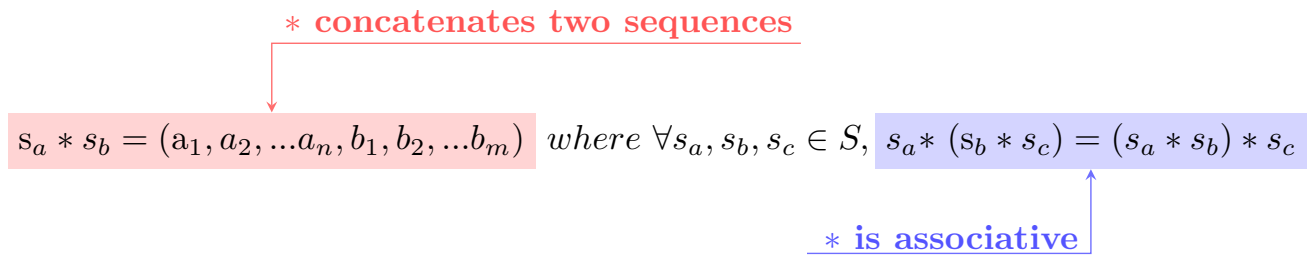

Definition 11. $\otimes$ is a sequence building operation $\otimes: G \times G \rightarrow G$. Take $s_{a}$ and $s_{b}$ as defined in Definition 10. Then $s_{a} \otimes s_{b}=\left\{\left(c_{1}, c_{2}, \ldots c_{m+n}\right)\right\}$, such that

$\otimes$ retains precedence relations in its input

$$
c_{i}= \begin{cases}a_{j} & \text { if } i>1 \text { and } a_{j-1} \in\left(c_{1} \ldots c_{i-1}\right) \\ b_{j} & \text { if } i>1 \text { and } b_{j-1} \in\left(c_{1} \ldots c_{i-1}\right) \\ a_{1} \text { or } b_{1} & \text { otherwise }\end{cases}
$$

The operator $*$ is simple concatenation. This operator is required because the temporal ordering of some actions must be specified. For example, if $h \in G$ represents the (sub)goal of obtaining hot water, then we must define $S_{h}=a * b$ due to the requirement that the kettle should be filled with water (action $a$ ) before it is turned on (action $b$ ). The temporal precedence relationship between these two actions requires an operator that yields strict sequential orders. Clearly, concatenation is not commutative: the sequence generated by $s_{a} * s_{b}$ is different from the output of $s_{b} * s_{a}$. Moreover, because $*$ generates sequences whose only structural relationship is precedence, it is associative: $s_{a} *\left(\mathrm{~s}_{b} * s_{c}\right)=\left(s_{a} * s_{b}\right) * s_{c}$. In sum, $(G, *)$ forms a monoid, which is an algebraic structure consisting of a set equipped with an operation that is closed, unital and associative (see Box 1).

The operator $\otimes$ generates sets of sequences whose orders vary, with the only constraint that the relative ordering within its arguments is retained. For example, for two sequences $s_{a}=(a, b, d), s_{b}=(c), s_{a} \otimes s_{b}=$ $\{(a, b, d, c),(a, b, c, d),(a, c, b, d),(c, a, b, d)\}$. In each of the sequences generated by $s_{a} \otimes s_{b}, a$ precedes $b$, which precedes $d$. So while $\otimes$ allows for flexibility in terms of the order of the actions in the sequences, the flexibility 
is constrained by the sequential properties of $s_{a}$, whose precedence relations must be retained in the output of $s_{a} \otimes s_{b}$. Because $\otimes$ is a sequence-building operator that is constrained only be the sequential properties of its input (i.e., the ordering within its input arguments), $\otimes$ is associative. But as it does not specify the ordering among its input arguments, $\otimes$ is also commutative. $(G, \otimes)$ therefore forms a commutative monoid (see Box 1).

The two notions of precedence and flexibility are combined in $(G, \otimes, *)$, which is an algebraic structure called a trace monoid (also called partially commutative monoid; see Box 1). A trace monoid is a monoid of traces, which are sets of sequences that form equivalence classes (Mazurkiewicz, 1995). In $(G, \otimes, *)$ the traces contain equivalent sequences generated by $\otimes$ and $*$. In a trace monoid, two sequences are equivalent if they only differ in the order of a pair of elements for which an independency relation is defined ${ }^{8}$. These independent elements are allowed to commute in the sequences of the equivalence class ${ }^{9}$. Consider the independency $I=\{(b, c),(c, b)\}$, which holds that the actions $b$ and $c$ are allowed to commute; no precedence relation between them is specified. Given $(b, c) \in I$, we say that two action sequences are equivalent if they differ only in the ordering of $b$ and $c$. The trace monoid is then said to contain a trace where $a c b \sim b c a$.

To sum up, defining the trace monoid $(G, \otimes, *)$ allows us to achieve simultaneously temporal precedence and temporal flexibility. The operator $*$ is required to build sequences where temporal precedence is necessary (e.g., 'grab milk' must precede 'pour milk into cup'), and $\otimes$ is used to generate action sequences whose temporal relationship is not specified (e.g., the action 'grab milk' can precede or follow 'put teabag in cup'). The combined use of $*$ and $\otimes$ leads to equivalence classes of sequences that contain a mixing of intermediate goals that are temporally independent of other intermediate goals. The mixing procedure introduced by $\otimes$ might destroy immediate precedence (or temporal adjacency) relationships in the output of $*$, but this is unproblematic: while it makes sense to let 'open fridge' be directly followed by 'grab milk', this is not necessary. One could open the fridge, perform all other tea-making preparations, and then grab the milk.

\subsubsection{Hierarchical relations between action sequences}

While the output of the two associative operators are sets of sequences, these sequences contain underlying structure if we take their derivational history into account (cf. 'configurational properties' in Miller et al., 1960). For instance, given the atomic actions in Figure 6, the sequence $(a, b, c, d)$ corresponds to the goal of making black tea. By itself, this sequence does not provide a lot of information about the precedence relations that might hold for the complex action; it could in principle have been generated via $(((a \otimes b) \otimes c) \otimes d)$. Such information can be inferred only if additional action sequences are observed that achieve the same goal (see Box 3). Knowing how the sequence was derived allows us to specify the temporal constraints to which the action sequence must adhere, which in turn provides information about the causal structure of the action plan. Thus, by deriving $[(a, b, c, d)] \in(a * b) \otimes(c * d)$, we make temporal precedence relations concrete: $a$ must precede $b$, and $c$ must precede $d$.

The derivational history of the sequence provides information about which other sequences are also possible. From observing only $(a, b, c, d)$, it would be impossible to know whether $(c, a, b, d)$ is also a fine sequence. However, that knowledge can be deduced if we know how the sequence was derived, because $(a * b) \otimes(c * d)$ also generates the sequence $(c, a, b, d)$. The derivational history thus provides information that is not present in the temporal structure of the sequence, including information about the relationship between the output sequence and its subsequences. In $(c, a, b, d)$, it is still the case that $c$ precedes $d$, even though they are not adjacent anymore. But by taking into account the derivational steps leading to $(c, a, b, d)$, we can specify a hierarchical relationship between $(c, d)$ and $(c, a, b, d)$, which states that $(c, d) \in(c, a, b, d)$ because $(c, a, b, d)$ was generated via $(a, b) \otimes(c, d)$. This relationship holds even though $(c, d)$ is not a subsequence of $(c, a, b, d)$. Action sequences can be seen as hierarchical sequences, which are sequences with a derivational history that specifies how they relate to the action sequences from which they are derived. This allows us to go beyond the sequential structure of actions in a system that is still weakly compositional.

\footnotetext{
${ }^{8}$ Independency relations are symmetric (i.e., if $(a, b)$ is present, then so is $(b, a)$ ) and irreflexive (i.e., there are no relations of type $(a, a)$ ), and can be extended to relations between sequences (see Mazurkiewicz, 1995).

${ }^{9}$ Commutativity in the general sense is slightly different from the way it is used in the context of traces. In the general sense (as used in Box 1), it refers to an operation which produces the same output if the order of the operands is changed, such as in $a \otimes b=b \otimes a$. In the context of a trace monoid, the notion of sameness is replaced by equivalence, where $a \otimes b=\{a b, b a\}$, and $a b \neq b a$ but $a b \sim b a$.
} 


\section{Box 3. Inferring plans from action sequences}

In order to achieve a given goal, the relative order of some related actions must be specified, whereas that of some unrelated actions can be left undefined. Given a set of observed action sequences that successfully reach the same goal, the abstract plan to reach that goal can be extracted via the intersection of the sets of binary relations representing the sequences.

As a simple illustration, consider a sequence $x=(a, b, c, d)$ consisting of non-repeating atomic actions. The precedence relations for $x$ are $a \prec b \prec c \prec d$. The sequence can be represented as a set of binary relations. If we take these binary relations to represent precedence, $x$ will be represented as $\{(a, b),(b, c),(c, d),(a, d),(a, c),(b, d)\}$. By observing only $x$, it is not immediately clear which of these elements are dependent and which are not. However, observing the sequence $y=(c, a, b, d)$ (represented as $y=\{(c, a),(a, b),(b, d),(c, d),(c, b),(a, d)\})$, which achieves the same goal, provides more information. The plan to reach the goal is represented by the intersection of the sets of binary relations:

$$
x \cap y=\{(a, b),(c, d),(b, d)\}
$$

This intersection corresponds to the plan of making black tea (see Figure 6). Notice how this partial order is compatible with the previously unseen sequence $(a, c, b, d)$, which reaches the same goal successfully as well.

\section{Language vs. action}

\subsection{A formal comparison}

In the previous sections we described the properties of hierarchical linguistic structure (generated by $\oplus$ ) using a magma. When this formalism was applied to actions, it appeared to be too strong, deriving multiple 'interpretations' from unambiguous action sequences, and too weak, as it does not generate temporally ordered structures. To overcome these limitations, our alternative formalism of actions described action structures as a trace monoid (generated by non-commutative $*$ and commutative $\otimes$ ). A crucial difference between these algebraic structures (see Box 1) is that the operation associated with magmas is non-associative, whereas that associated with monoids is associative. As a consequence, the structure generated by $\oplus$ and represented in the magma is strongly compositional: the constituent structure of the input to $\oplus$ is retained in its output. This is important because both syntactic operations and semantic interpretation are structure-dependent. If $\oplus$ had been associative, the internal structure of each combination would be lost, so syntactic rules could not target constituents. Moreover, as meaning could not be derived from constituent structure, sentences could not be structurally ambiguous; the system would generate only one output for ((deep $\oplus$ blue) $\oplus$ sea) and (deep $\oplus$ (blue $\oplus$ sea)). In contrast, the action structure generated by the operators $*$ and $\otimes$ and represented in the trace monoid is weakly compositional. This weakly compositional, order-sensitive model can account for the relevant properties of action structures.

\subsection{The nature of structure}

Our formal characterizations of language and action show that their structural representations have different properties, in particular concerning the relevance of constituency (for a similar conclusion from the neuroimaging literature, see Papitto et al., 2020 and Zaccarella et al., 2021). The same conclusion is reached when we compare language and action in terms of the properties of syntactic structure discussed in Section 2.1 .

1. Unbounded. has been argued that the combinatorial operation involved in building syntactic structures evolved from pre-existing systems for tool use, also called "Action Merge" (Fujita, 2014, 2017). This operation is thought to apply recursively (Fujita, 2017; Pulvermüller, 2014; Stout \& Chaminade, 2009), even though Action Merge is bounded (Fujita, 2014). A distinctive feature of recursively generated hierarchical output is self-similarity across levels: recursively generated structures are characterized by self-embedding of 'tokens' of the same 'type' (Martins, 2012). A first step would therefore be to 
examine whether action hierarchies are self-similar. However, that requires that we know what the types are. Consider the structure in Figure 5. One could combine 'open fridge' and 'grab milk' into an action constituent, which could be labeled 'get milk'. Here, it is unclear whether 'get milk' is of the same type as 'grab milk'. Moreover, it seems plausible that the action 'pour hot water into cup' is similar to 'pour milk into cup', but that is because the tokens are similar (both involve pouring), not necessarily because their types are. Without a theoretical specification of the types of actions, it cannot be determined whether actions are recursively generated.

Our primary goal is to evaluate the claim that the hierarchical structures found in language and action are analogous. The validity of this claim rests on positive evidence that actions, like language, are recursively generated. In the absence of such evidence, it is premature to conclude that actions are structurally analogous to language.

2. Endocentric. Some of the hierarchical representations of actions that are proposed in the literature contain action constituents with one key element, or 'head', which performs the core of the action and determines its (end)goal (e.g., Jackendoff, 2007, 2009, 2011; Fischmeister et al., 2017). While this makes the structures 'headed', it does not make them endocentric. That is, it seems that this head merely serves to describe the main action of the action sequence, rather than to provide a label for the constituent it is dominated by. In Figure 5, for instance, the action constituent formed by the combination of 'open fridge' and 'grab milk' is not a type of either of these actions. In line with the idea that endocentricity is unique to language (Boeckx, 2009; Hornstein, 2009), action hierarchies seem to be exocentric.

A plausible reason for the difficulty in assigning labels to action constituents is that actions do not have clear conceptual units, such as words (Moro, 2014a; Berwick et al., 2011), and that groups of actions do not obligatorily fall into a closed set of distinguishable categories, such as NP or VP (Jackendoff \& Pinker, 2005). Without these categories, groupings of actions into constituents cannot be labeled or 'syntactically named', which means that there are no grammatical constraints on how the resulting constituents can be used in further combinations.

3. Unordered. Representations of actions are intimately tied to the physical environment in which the actions are performed (Graves, 1994; Kuperberg, 2020; Moro, 2014a; Zaccarella et al., 2021). As such, they are not order-independent: some sub-actions must precede others in order for the action to achieve its goal (Fitch \& Martins, 2014), and indeed, the output of Action Merge is inherently ordered $(\text { Fujita, 2014) })^{10}$. Comparing this to language, we see that the externalization of spoken language is also sequential, but that sequential order does not play a role in the representation of syntactic relations, which are invariably structure-dependent.

It has been proposed that closely related actions, which can be separated by arbitrarily many 'embedded' actions (e.g., [open door [switch on light [brush teeth] switch off light] close door]), are similar to long-distance dependencies in language (Pulvermüller \& Fadiga, 2010; Pulvermüller, 2014). This analogy is incorrect, however, because long-distance dependencies are related to the hierarchical organization of constituent structure. These action dependencies, instead, have serial and temporal properties: you cannot close a door before having opened it (Dominey et al., 2003; Moro, 2015; Zaccarella et al., 2021). If they were truly hierarchical, the embedded action would be expected to adhere to structural restrictions on its distribution, which would be the case if the embedding of [brush teeth] at a different position, like in [open door [brush teeth] [switch on light switch off light] close door], were not allowed. Moreover, if the dependency were hierarchical, it should not be affected by linearly or temporally intervening actions, so whatever happens during [brush teeth] should not be able to affect the action [switch off light]. As neither appears to be the case, it is more appropriate to label the dependency between two actions temporal (or causal) rather than hierarchical (Moro, 2014b, 2015). Indeed, actions and events can be understood in terms of temporal (and causal) structure (McRae et al., 2019; Zacks \& Tversky, 2001), and oddly ordered complex actions, which are thought of as ungrammatical actions

\footnotetext{
${ }^{10}$ Even under an analysis in which immediate precedence plays a role in syntax (as in Kayne, 2011), the crucial difference between language and actions remains: if two linguistic objects $\alpha$ and $\beta$ are not adjacent in their base-generated position (i.e., they do not form the ordered pair $\langle\alpha, \beta\rangle$ ), their relationship is defined as a relationship that refers to the (hierarchical) constituents they are contained in, not as a relationship that refers to their linear or temporal order. There is no such constraint in actions, where some actions must precede (distant) others, regardless of the relationship between the action constituents in which they are contained.
} 
(e.g., Maffongelli et al., 2019), reflect the violation of 'temporal rules' rather than phrase-structure rules (Zaccarella et al., 2021).

A plausible reason for the observation that none of the properties of hierarchy in syntax are found in actions is that the analogy between language and action is not to be found in syntactic structure, but rather in conceptual structure (Jackendoff, 2007; Zaccarella et al., 2021). An important difference is that syntax is computationally autonomous, having its own principles and properties that cannot be reduced to other factors, such as meaning (Adger, 2018; Berwick, 2018; Chomsky, 1957). The application of these principles is constrained by economy conditions (e.g., locality, minimality; see Collins, 2001), but not by whether they generate interpretable output. Therefore, in language there is an independent notion of grammaticality: sentences are ungrammatical if their structures cannot be generated by the rules of syntax, or if they violate conditions on these rules. One way to illustrate this is by means of interpretable but nevertheless ungrammatical sentences. A sentence such as "which boy did they meet the girl who insulted?" is ungrammatical but can be interpreted (i.e., corresponding to the logical statement "for which $\mathrm{x}$, $\mathrm{x}$ a boy, did they meet the girl who insulted x?"). Its deviance is due to the violation of a purely formal (locality) principle constraining the grammar, which is unrelated to its semantic interpretability. Conversely, the sentence "colorless green ideas sleep furiously" is semantically odd, yet fully grammatical (Adger, 2018; Berwick, 2018; Chomsky, 1957), showing that grammaticality does not boil down to meaningfulness or interpretability.

In contrast, the validity of action sequences seems related to their coherence, in terms of both logical consistency and environmental appropriateness. It has been suggested that a complex action is 'ungrammatical' or 'ill-formed' if its sub-parts are ordered in such a way that the action's overall goal cannot be achieved (Jackendoff, 2007; Maffongelli et al., 2019). The 'grammaticality' of an action is thus intimately tied to the fulfillment of its goal, showing that the notion 'ungrammatical' is very different for action sequences and sentences (Graves, 1994; Zaccarella et al., 2021). On this interpretation, an 'ungrammatical' action is similar to a sentence which does not convey the intended meaning, either because it is logically incoherent or because it is situationally inappropriate. The action equivalent of a logically incoherent sentence could be an action sequence in which a coffee grinder is turned on before the coffee beans are added. This is logically incoherent because it violates causality principles of the physical environment. An action like turning off the light when walking into your office during nighttime, instead, does not violate such constraints, but it would be situationally inappropriate because it would preclude you from seeing anything (e.g., Reason, 1979).

Because there is no autonomous action syntax, there is no independent notion of grammaticality, devoid of goal-dependent meaning. As a result, it is unclear how to evaluate whether a given structural decomposition of complex actions into constituents is veridical if we do not know the goal or general conceptual content of the action (Berwick \& Chomsky, 2017; Jackendoff, 2007). It seems that the decomposition of an action sequence into a hierarchical tree structure only works to the extent that the subactions are meaningful or coherent (i.e., represent subgoals).

\subsection{Levels of abstraction}

The difference between language and actions in terms of their dependence on hierarchical and sequential structure can be captured quite naturally under the distinction between hierarchical sequences and hierarchical sets, a terminological contrast adopted by Fitch and Martins (2014) to distinguish possible interpretations of the term hierarchy ${ }^{11}$. Fitch and Martins (2014) describe hierarchical sets as structures that specify the superior/inferior relation between their elements (i.e., specifying containment), but whose elements are unordered at any given level. Hierarchical sequences, instead, are hierarchical structures in which sequential order matters: at least some elements at any given level represent a sequence rather than a set.

We will argue that language needs to be described in terms of both hierarchical sequences and hierarchical sets, but that actions can be described as hierarchical sequences only (see Figure 8). Regarding language, the mapping between the sequential order of words and their hierarchical organization into constituents represents the level of hierarchical sequences (i.e., the interface between hierarchical structure and linear order; see middle panel, top row in Figure 8). In this hierarchically structured sequence, we can describe a speaker's knowledge about linearized properties of their language (e.g., word order, morphosyntax), such

\footnotetext{
${ }^{11} \mathrm{~A}$ similar distinction is emphasized by Tettamanti and Moro (2012), who discuss the different meanings of 'hierarchical organization' in terms of sequential vs. internal hierarchy, describing respectively the computation of sequential hierarchical information (externalized) and the computation of non-linear hierarchical relations (mind-internal).
} 
as whether heads precede or follow their dependents (e.g., English vs. Japanese). Hierarchical sets are further abstractions from these hierarchical sequences, which are not realized in the physical properties of the linguistic signal (left panel, top row in Figure 8). This level is explanatorily relevant for syntactic theory because it naturally captures the properties of syntax described in Section 2.1: hierarchical sets generated by Merge are unbounded, endocentric, and unordered (Lasnik, 2000). Therefore, at this level, we can account for both structural relations within languages and structural generalizations between languages (e.g., the head-dependent relations in English and Japanese are identical at the level of hierarchical sets).

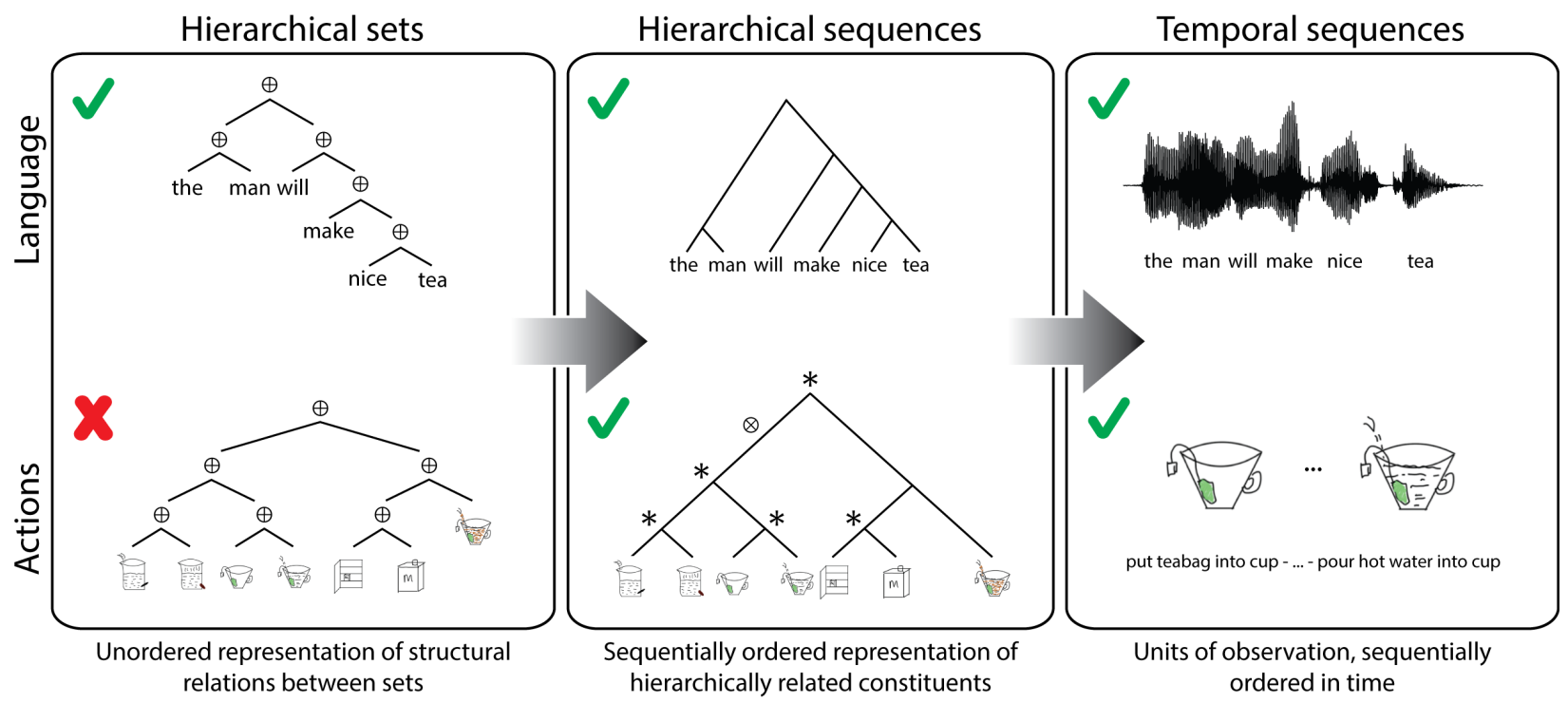

Figure 8: Three representational levels to describe hierarchically structured sequential information. The levels become increasingly concrete, from what is abstractly represented (on the left) to what is physically observed (on the right). The symbol at each node in the hierarchical structures indicates which operator was used to combine the elements, thus representing the derivational history of the sets (left) or sequences (middle).

As we noted in Section 3.2.3, actions might be seen as ordered sequences of events that are hierarchically structured (middle panel, bottom row of Figure 8). However, the structural properties of action sequences cannot be described in a way which has completely abstracted away from the physical instantiation of the action sequence, most clearly because the (representation of the) hierarchical structure of action sequences contains information about temporal order. Because the properties of syntactic structure are not found in actions, we do not have to postulate hierarchical sets as an explanatorily or functionally relevant level of abstraction for actions.

The distinction between hierarchical sets and sequences is useful in explaining why it has been found that the brain areas involved in language processing (in particular, Brodmann's area 44 in the left inferior frontal gyrus) are also activated in response to tasks involving hierarchically organized actions (e.g., Higuchi et al., 2009; Koechlin \& Jubault, 2006). These results support the idea that there is a supramodal hierarchical processor in the brain which processes the hierarchical structures of cognitive systems such as language and action (Fadiga et al., 2009; Fazio et al., 2009; Fiebach \& Schubotz, 2006; Higuchi et al., 2009; Jeon, 2014; Koechlin \& Jubault, 2006; Tettamanti \& Weniger, 2006). Crucially, however, instead of describing complex actions in terms of non-linear relations defined over hierarchical structures, these accounts refer to the processing of sequences which happen to have hierarchical structure (for a related discussion, see Martins et al., 2019 and Zaccarella et al., 2021). The overlapping activation patterns for language and action might therefore point not to shared brain regions processing hierarchical, non-linear relations (operating over hierarchical sets), but rather to shared brain regions implicated in the linearization of hierarchically structured information (i.e., hierarchical sequences; see also Boeckx et al., 2014; Matchin \& Hickok, 2020; Uddén \& Bahlmann, 2012). To account for the former process, a reconceptualization of how linguistic representations might be coded in neural systems is likely needed (Martin, 2016, 2020; Martin \& Doumas, 2017; Meyer et al., 
2019). We believe that a fruitful avenue for further investigation into the relationship between language and action concerns the externalization of hierarchically organized information into structured sequences rather than the (generation of the) hierarchical structure itself. The overlap between language and action then has to do with the fact that, externally, both are structured sequences, even though their internal structures are quite different.

\section{Conclusions}

In response to the claim that language and action are analogous because they are both organized hierarchically, we argued in this paper that the formal properties of hierarchy in both domains are fundamentally different. Our main argument is that the language system can embody strong compositionality, as both syntactic rules and semantic interpretation are structure-dependent. Structural analyses in language are thus concerned with non-terminal nodes in the hierarchical structure of syntax. Actions, instead, are weakly compositional: regularities in action structures are dependent on the temporal order of the atomic actions, not on their hierarchical organization into action goals. Analyses of actions are thus concerned with terminal nodes in the action hierarchy. Based on this difference, we argue that the structure of syntax is best described as a system of hierarchical sets, whereas action structures can be described as hierarchical sequences.

In order to formally capture the strong compositionality of language, we described the algebraic structure corresponding to the ordered set of hierarchical structures in language as a magma, whose non-associative combinatorial operator was defined as binary set formation. This set-based formalism integrates the three properties of syntactic structure (i.e., unboundedness, endocentricity, and unorderedness) with the description of syntax as a system of hierarchical sets and the fact that language exhibits strong compositionality. When this model was applied to actions, it appeared to be both too strong (i.e., it makes structural distinctions which should not be made) and too weak (i.e., it does not capture the importance of temporal precedence). We therefore proposed an alternative model for actions, which used two sequence building operators that organize actions by sequential relations. This yielded an ordered set of action structures that could be described as a trace monoid. The associativity of the two operators illustrates how actions exhibit a weaker form of compositionality, which is based on sequential rather than hierarchical structure. This aligns well with our argument that actions are best described in terms of hierarchical sequences. In sum, the formal tools needed to describe language are fundamentally different from those required to the describe the action system. We believe that this result has important implications not only for comparative cognitive science but also for cognitive neuroscience, as it points to differences in the ways in which hierarchies are represented in the brain.

\section{Glossary}

- Compositionality: Property of a system which holds that the meaning of a complex expression is built up from the meanings of its parts and the way in which they are combined.

- C-command: Structural relation between nodes in a hierarchical structure. Node $\alpha$ c-commands a node $\beta$ iff $\beta$ is (contained in) the sister node of $\alpha$.

- Endocentricity: Property of syntactic structures. A structural combination is endocentric if it fulfills the same grammatical function as one of its parts. Endocentric structures are contrasted with exocentric structures, in which the grammatical function of the combination is not the same as that of its parts.

- Equivalence class: A subset (of a set) containing elements that are related under the equivalence relation. An equivalence class is denoted by square brackets: $[a]=\{b \mid b \sim a\}$.

- Equivalence relation: An equivalence relation on a set is a binary relation that is reflexive, symmetric, and transitive. An equivalence relation is indicated by $\sim$, so $a \sim b$ denotes that $a$ is equivalent to $b$.

- Hasse diagram: A mathematical diagram used to visualize an ordered set.

- Linear Correspondence Axiom: An algorithm that maps hierarchical structure onto linear order using the c-command relation. 
- Merge: Combinatorial procedure to generate syntactic structure, formally defined as binary set formation: $\operatorname{Merge}(\alpha, \beta)=\{\alpha, \beta\}$.

- Magma: An algebraic structure consisting of a set equipped with a binary operation which is closed and not associative.

- Monoid: An algebraic structure consisting of a set equipped with a binary operation that is closed, unital and associative.

- Partial order: A set with a binary relation that is transitive and antisymmetric.

- Partition: A partition of a set $S$ is a set of non-empty subsets of $S$ that cover $S$ and are pairwise disjoint.

- Recursion: Property of a function, which lets it take its output as its input.

- Surjective: Mathematical property of a function $f: X \rightarrow Y$ which holds that for every element $y \in Y$ there exists at least one element $x \in X$ such that $f(x)=y$.

- Total order: A set with a binary relation that is transitive, antisymmetric, and total.

- Trace monoid: A monoid whose operation is partially commutative.

\section{Acknowledgements}

We thank Giosuè Baggio, Cedric Boeckx, Helen de Hoop, and Bob van Tiel for helpful comments on earlier versions of this work. We also thank Steven Phillips for help with aspects of the formal work. For annotating the equations in our formal work we used the code from https://github.com/synercys/annotated_latex _equations. Andrea E. Martin was supported by a Max Planck Research Group and a Lisa Meitner Research Group "Language and Computation in Neural Systems" from the Max Planck Society and by the Netherlands Organisation (NWO) for Scientific Research (grant 016.Vidi.188.029). 


\section{References}

Adger, D. (2018). The autonomy of syntax. In N. Hornstein, H. Lasnik, P. Patel-Grosz, \& C. Yang (Eds.), Syntactic Structures after 60 Years: The Impact of the Chomskyan Revolution in Linguistics (pp. 153-175). De Gruyter Mouton.

Badre, D. (2008). Cognitive control, hierarchy, and the rostro-caudal organization of the frontal lobes. Trends in Cognitive Sciences, 12(5), 193-200. doi: 10.1016/j.tics.2008.02.004

Baggio, G. (2021). Compositionality in a parallel architecture for language processing. Cognitive Science, 45(5), e12949. doi: $10.1111 / \operatorname{cogs} .12949$

Berwick, R. C. (2018). Revolutionary new ideas appear infrequently. In N. Hornstein, H. Lasnik, P. PatelGrosz, \& C. Yang (Eds.), Syntactic Structures after 60 Years: The Impact of the Chomskyan Revolution in Linguistics (pp. 177-194). De Gruyter Mouton.

Berwick, R. C., \& Chomsky, N. (2017). Why only us: Recent questions and answers. Journal of Neurolinguistics, 43, 166-177. doi: 10.1016/j.jneuroling.2016.12.002

Berwick, R. C., Okanoya, K., Beckers, G. J. L., \& Bolhuis, J. J. (2011). Songs to syntax: The linguistics of birdsong. Trends in Cognitive Sciences, 15(3), 113-121. doi: 10.1016/j.tics.2011.01.002

Bloom, P. (1994). Generativity within language and other cognitive domains. Cognition, 51(2), 177-189. doi: 10.1016/0010-0277(94)90014-0

Boeckx, C. (2009). The nature of Merge: Consequences for language, mind, and biology. In M. PiattelliPalmarini, J. Uriagereka, \& P. Salaburu (Eds.), Of Minds and Language: A Dialogue with Noam Chomsky in the Basque Country (pp. 44-57). Oxford: Oxford University Press.

Boeckx, C., \& Fujita, K. (2014). Syntax, action, comparative cognitive science, and Darwinian thinking. Frontiers in Psychology, 5. doi: 10.3389/fpsyg.2014.00627

Boeckx, C., Martinez-Alvarez, A., \& Leivada, E. (2014). The functional neuroanatomy of serial order in language. Journal of Neurolinguistics, 32, 1-15. doi: 10.1016/j.jneuroling.2014.07.001

Botvinick, M. M. (2008). Hierarchical models of behavior and prefrontal function. Trends in Cognitive Sciences, 12(5), 201-208. doi: 10.1016/j.tics.2008.02.009

Chomsky, N. (1957). Syntactic Structures. The Hague: Mouton de Gruyter.

Chomsky, N. (1959). A Review of B. F. Skinner's Verbal Behavior. Language, 35(1), 26-58. doi: 10.2307/ 411334

Chomsky, N. (1995a). Bare phrase structure. In H. R. Campos \& P. M. Kempchinsky (Eds.), Evolution and Revolution in Linguistic Theory: Essays in Honor of Carlos Otero (pp. 51-109). Washington, DC: Georgetown University Press.

Chomsky, N. (1995b). The Minimalist Program. Cambridge, MA: MIT Press.

Chomsky, N. (2013). Problems of projection. Lingua, 130, 33-49. doi: 10.1016/j.lingua.2012.12.003

Collins, C. (2001). Economy conditions in syntax. In M. Baltin \& C. Collins (Eds.), The Handbook of Contemporary Syntactic Theory (pp. 45-61). Oxford: Blackwell Publishers.

Collins, C. (2017). Merge(X,Y) $=\{\mathrm{X}, \mathrm{Y}\}$. In L. Bauke \& A. Blümel (Eds.), Labels and Roots (pp. 47-68). De Gruyter Mouton. doi: 10.1515/9781501502118-003

Cooper, R. P., \& Shallice, T. (2000). Contention scheduling and the control of routine activities. Cognitive Neuropsychology, 17(4), 297-338. doi: 10.1080/026432900380427

Cooper, R. P., \& Shallice, T. (2006). Hierarchical schemas and goals in the control of sequential behavior. Psychological Review, 113(4), 887. doi: 10.1037/0033-295X.113.4.887

Corballis, M. C. (1991). The Lopsided Ape: Evolution of the Generative Mind. Oxford: Oxford University Press.

de Waal, F. B. M., \& Ferrari, P. F. (2010). Towards a bottom-up perspective on animal and human cognition. Trends in Cognitive Sciences, 14(5), 201-207. doi: 10.1016/j.tics.2010.03.003

Dominey, P. F., Hoen, M., Blanc, J.-M., \& Lelekov-Boissard, T. (2003). Neurological basis of language and sequential cognition: Evidence from simulation, aphasia, and ERP studies. Brain and Language, 86(2), 207-225. doi: 10.1016/S0093-934X(02)00529-1

Everaert, M. B. H., Huybregts, M. A. C., Chomsky, N., Berwick, R. C., \& Bolhuis, J. J. (2015). Structures, not strings: Linguistics as part of the cognitive sciences. Trends in Cognitive Sciences, 19(12), 729-743. doi: $10.1016 /$ j.tics.2015.09.008 
Fadiga, L., Craighero, L., \& D'Ausilio, A. (2009). Broca's Area in language, action, and music. Annals of the New York Academy of Sciences, 1169(1), 448-458. doi: 10.1111/j.1749-6632.2009.04582.x

Fazio, P., Cantagallo, A., Craighero, L., D'Ausilio, A., Roy, A. C., Pozzo, T., ... Fadiga, L. (2009). Encoding of human action in Broca's area. Brain, 132(7), 1980-1988. doi: 10.1093/brain/awp118

Fiebach, C. J., \& Schubotz, R. I. (2006). Dynamic anticipatory processing of hierarchical sequential events: A common role for broca's area and ventral premotor cortex across domains? Cortex, 42(4), 499-502. doi: 10.1016/S0010-9452(08)70386-1

Fischmeister, F. P., Martins, M. J. D., Beisteiner, R., \& Fitch, W. T. (2017). Self-similarity and recursion as default modes in human cognition. Cortex, 97, 183-201. doi: 10.1016/j.cortex.2016.08.016

Fitch, W. T. (2010). Three meanings of "recursion": Key distinctions for biolinguistics. In H. Yamakido, R. K. Larson, \& V. Déprez (Eds.), The Evolution of Human Language: Biolinguistic Perspectives (pp. 73-90). Cambridge: Cambridge University Press. doi: 10.1017/CBO9780511817755.005

Fitch, W. T., \& Martins, M. D. (2014). Hierarchical processing in music, language, and action: Lashley revisited. Annals of the New York Academy of Sciences, 1316(1), 87-104. doi: 10.1111/nyas.12406

Fujita, K. (2014). Recursive Merge and human language evolution. In T. Roeper \& M. Speas (Eds.), Recursion: Complexity in Cognition (pp. 243-264). Springer.

Fujita, K. (2017). On the parallel evolution of syntax and lexicon: A Merge-only view. Journal of Neurolinguistics, 43, 178-192. doi: 10.1016/j.jneuroling.2016.05.001

Fukui, N. (2011). Merge and Bare phrase structure. In C. Boeckx (Ed.), The Oxford Handbook of Linguistic Minimalism. Oxford: Oxford University Press. doi: 10.1093/oxfordhb/9780199549368.013.0004

Fukui, N., \& Zushi, M. (2004). Introduction. In N. Chomsky (Ed.), The Generative Enterprise Revisited: Discussions with Riny Huybregts, Henk van Riemsdijk, Naoki Fukui and Mihoko Zushi (pp. 1-25). Berlin: Walter de Gruyter.

Graves, P. (1994). Flakes and ladders: What the archaeological record cannot tell us about the origins of language. World Archaeology, 26 (2), 158-171. doi: 10.1080/00438243.1994.9980270

Greenfield, P. M. (1991). Language, tools and brain: The ontogeny and phylogeny of hierarchically organized sequential behavior. Behavioral and Brain Sciences, 14 (4), 531-551. doi: 10.1017/S0140525X00071235

Guest, O., \& Martin, A. E. (2021). How computational modeling can force theory building in psychological science. Perspectives on Psychological Science, 1-14. doi: 10.1177/1745691620970585

Hauser, M. D., Chomsky, N., \& Fitch, W. T. (2002). The faculty of language: What is it, who has it, and how did it evolve? Science, 298(5598), 1569-1579. doi: 10.1126/science.298.5598.1569

Hauser, M. D., Yang, C., Berwick, R. C., Tattersall, I., Ryan, M. J., Watumull, J., .. Lewontin, R. C. (2014). The mystery of language evolution. Frontiers in Psychology, 5. doi: 10.3389/fpsyg.2014.00401

Higuchi, S., Chaminade, T., Imamizu, H., \& Kawato, M. (2009). Shared neural correlates for language and tool use in Broca's area. NeuroReport, 20(15), 1376-1381. doi: 10.1097/WNR.0b013e3283315570

Holloway, R. L. (1969). Culture: A human domain. Current Anthropology, 10, 395-412.

Hornstein, N. (2009). A Theory of Syntax: Minimal Operations and Universal Grammar. Cambridge: Cambridge University Press.

Hornstein, N. (2017). On Merge. In J. McGilvray (Ed.), The Cambridge Companion to Chomsky (Second ed., pp. 69-86). Cambridge: Cambridge University Press. doi: 10.1017/9781316716694.004

Humphreys, G. W., \& Forde, E. M. E. (1998). Disordered action schema and action disorganisation syndrome. Cognitive Neuropsychology, 15(6/7/8), 771-811.

Jackendoff, R. (1977). X' Syntax: A Theory of Phrase Structure. Cambridge, MA: MIT Press.

Jackendoff, R. (2002). Foundations of Language: Brain, Meaning, Grammar, Evolution. Oxford: Oxford University Press.

Jackendoff, R. (2007). Language, Consciousness, Culture: Essays on Mental Structure. Cambridge, MA: MIT Press.

Jackendoff, R. (2009). Parallels and nonparallels between language and music. Music Perception, 26(3), 195-204. doi: 10.1525/mp.2009.26.3.195

Jackendoff, R. (2011). What is the human language faculty? Two views. Language, 87(3), 586-624.

Jackendoff, R., \& Pinker, S. (2005). The nature of the language faculty and its implications for evolution of language (Reply to Fitch, Hauser, and Chomsky). Cognition, 97(2), 211-225. doi: 10.1016/j.cognition .2005 .04 .006 
Jeon, H.-A. (2014). Hierarchical processing in the prefrontal cortex in a variety of cognitive domains. Frontiers in Systems Neuroscience, 8. doi: 10.3389/fnsys.2014.00223

Joshi, A. K., \& Schabes, Y. (1997). Tree-Adjoining Grammars. In G. Rozenberg \& A. Salomaa (Eds.), Handbook of Formal Languages: Volume 3 Beyond Words (pp. 69-123). Berlin, Heidelberg: Springer. doi: 10.1007/978-3-642-59126-6_2

Kayne, R. S. (1994). The Antisymmetry of Syntax. Cambridge, MA: MIT Press.

Kayne, R. S. (2011). Why are there no directionality parameters? In Proceedings of WCCFL (Vol. 28, pp. $1-23)$.

Koechlin, E., \& Jubault, T. (2006). Broca's area and the hierarchical organization of human behavior. Neuron, 50(6), 963-974. doi: 10.1016/j.neuron.2006.05.017

Kuperberg, G. R. (2020). Tea with milk? A hierarchical generative framework of sequential event comprehension. Topics in Cognitive Science, 13(1), 256-298. doi: 10.1111/tops.12518

Lashley, K. S. (1951). The problem of serial order in behavior. In L. A. Jeffress (Ed.), Cerebral mechanisms in behavior (pp. 112-131). New York, NY: Wiley.

Lasnik, H. (2000). Syntactic Structures Revisited: Contemporary Lectures on Classic Transformational Theory. Cambridge, MA: MIT Press.

Maffongelli, L., D'Ausilio, A., Fadiga, L., \& Daum, M. M. (2019). The ontogenesis of action syntax. Collabra: Psychology, 5(1), 21. doi: 10.1525/collabra.215

Marcus, G. F. (2006). Cognitive architecture and descent with modification. Cognition, 101(2), 443-465. doi: $10.1016 /$ j.cognition.2006.04.009

Martin, A. E. (2016). Language processing as cue integration: Grounding the psychology of language in perception and neurophysiology. Frontiers in Psychology, 7. doi: 10.3389/fpsyg.2016.00120

Martin, A. E. (2020). A compositional neural architecture for language. Journal of Cognitive Neuroscience, 32(8), 1407-1427. doi: 10.1162/jocn_a_01552

Martin, A. E., \& Doumas, L. A. A. (2017). A mechanism for the cortical computation of hierarchical linguistic structure. PLOS Biology, 15(3), e2000663. doi: 10.1371/journal.pbio.2000663

Martins, M. D. (2012). Distinctive signatures of recursion. Philosophical Transactions of the Royal Society B: Biological Sciences, 367(1598), 2055-2064. doi: 10.1098/rstb.2012.0097

Martins, M. D., Bianco, R., Sammler, D., \& Villringer, A. (2019). Recursion in action: An fMRI study on the generation of new hierarchical levels in motor sequences. Human Brain Mapping, 40 (9), 2623-2638. doi: $10.1002 / \mathrm{hbm} .24549$

Matchin, W., \& Hickok, G. (2020). The cortical organization of syntax. Cerebral Cortex, 30(3), 1481-1498. doi: $10.1093 /$ cercor/bhz180

Mazurkiewicz, A. (1995). Introduction to Trace Theory. In V. Diekert \& G. Rozenberg (Eds.), The Book of Traces (pp. 3-41). Singapore: World Scientific. doi: 10.1142/9789814261456_0001

McRae, K., Brown, K. S., \& Elman, J. L. (2019). Prediction-based learning and processing of event knowledge. Topics in Cognitive Science, 13(1), 206-223. doi: 10.1111/tops.12482

Meyer, L., Sun, Y., \& Martin, A. E. (2019). Synchronous, but not entrained: Exogenous and endogenous cortical rhythms of speech and language processing. Language, Cognition and Neuroscience, 35(9), 1089-1099. doi: 10.1080/23273798.2019.1693050

Miller, G. A., Galanter, E., \& Pribram, K. H. (1960). Plans and the Structure of Behavior. New York, NY: Holt, Rinehart and Winston.

Moro, A. (2014a). On the similarity between syntax and actions. Trends in Cognitive Sciences, 18(3), 109-110. doi: 10.1016/j.tics.2013.11.006

Moro, A. (2014b). Response to Pulvermüller: The syntax of actions and other metaphors. Trends in Cognitive Sciences, 18(5), 221. doi: 10.1016/j.tics.2014.01.012

Moro, A. (2015). The Boundaries of Babel: The Brain and the Enigma of Impossible Languages. Cambridge, MA: MIT Press.

Norman, D. A. (1981). Categorization of action slips. Psychological Review, 88(1), 1-15. doi: 10.1037/ 0033-295X.88.1.1

O'Donnell, T. J., Hauser, M. D., \& Fitch, W. T. (2005). Using mathematical models of language experimentally. Trends in Cognitive Sciences, 9(6), 284-289. doi: 10.1016/j.tics.2005.04.011

Pagin, P., \& Westerståhl, D. (2010). Compositionality I: Definitions and Variants. Philosophy Compass, 5(3), 250-264. doi: 10.1111/j.1747-9991.2009.00228.x 
Papitto, G., Friederici, A. D., \& Zaccarella, E. (2020). The topographical organization of motor processing: An ALE meta-analysis on six action domains and the relevance of Broca's region. NeuroImage, 206, 116321. doi: 10.1016/j.neuroimage.2019.116321

Partee, B. H. (1995). Lexical semantics and compositionality. In L. R. Gleitman \& M. Liberman (Eds.), Language: An invitation to cognitive science, Vol. 1, 2nd ed (pp. 311-360). Cambridge, MA, US: The MIT Press.

Partee, B. H., ter Meulen, A., \& Wall, R. E. (1993). Mathematical methods in linguistics (Vol. 30). Dordrecht: Kluwer Academic Publishers.

Pulvermüller, F. (2014). The syntax of action. Trends in Cognitive Sciences, 18(5), 219-220. doi: 10.1016/ j.tics.2014.01.001

Pulvermüller, F., \& Fadiga, L. (2010). Active perception: Sensorimotor circuits as a cortical basis for language. Nature Reviews Neuroscience, 11(5), 351-360. doi: 10.1038/nrn2811

Reason, J. T. (1979). Actions not as planned. In G. Underwood \& R. Stevens (Eds.), Aspects of Consciousness (pp. 67-90). London: Academic Press.

Reinhart, T. (1983). Anaphora and Semantic Interpretation. London: Croom Helm.

Rizzi, L. (2004). On the study of the language faculty: Results, developments, and perspectives. The Linguistic Review, 21(3-4), 323-344. doi: 10.1515/tlir.2004.21.3-4.323

Rizzi, L. (2013). Introduction: Core computational principles in natural language syntax. Lingua, 130, 1-13. doi: 10.1016/j.lingua.2012.12.001

Rosenbaum, D. A., Cohen, R. G., Jax, S. A., Weiss, D. J., \& van der Wel, R. (2007). The problem of serial order in behavior: Lashley's legacy. Human Movement Science, 26(4), 525-554. doi: 10.1016/ j.humov.2007.04.001

Saito, M., \& Fukui, N. (1998). Order in phrase structure and movement. Linguistic Inquiry, 29(3), 439-474. doi: $10.1162 / 002438998553815$

Schwartz, M. F. (2006). The cognitive neuropsychology of everyday action and planning. Cognitive Neuropsychology, 23(1), 202-221. doi: 10.1080/02643290500202623

Steedman, M. (2000). The Syntactic Process. Cambridge, MA: MIT press.

Steedman, M. (2002). Plans, affordances, and Combinatory Grammar. Linguistics and Philosophy, 25(5), 723-753. doi: 10.1023/A:1020820000972

Stout, D., \& Chaminade, T. (2009). Making tools and making sense: Complex, intentional behaviour in human evolution. Cambridge Archaeological Journal, 19(1), 85-96. doi: 10.1017/S0959774309000055

Tettamanti, M., \& Moro, A. (2012). Can syntax appear in a mirror (system)? Cortex, 48(7), 923-935. doi: 10.1016/j.cortex.2011.05.020

Tettamanti, M., \& Weniger, D. (2006). Broca's area: A supramodal hierarchical processor? Cortex, 42(4), 491-494. doi: 10.1016/S0010-9452(08)70384-8

Uddén, J., \& Bahlmann, J. (2012). A rostro-caudal gradient of structured sequence processing in the left inferior frontal gyrus. Philosophical Transactions of the Royal Society B: Biological Sciences, 367(1598), 2023-2032. doi: 10.1098/rstb.2012.0009

Uithol, S., van Rooij, I., Bekkering, H., \& Haselager, P. (2012). Hierarchies in action and motor control. Journal of Cognitive Neuroscience, 24(5), 1077-1086. doi: 10.1162/jocn_a_00204

van Rooij, I., \& Blokpoel, M. (2020). Formalizing verbal theories. Social Psychology, 51(5), 285-298. doi: $10.1027 / 1864-9335 / \mathrm{a} 000428$

Watumull, J., Hauser, M. D., Roberts, I. G., \& Hornstein, N. (2014). On recursion. Frontiers in Psychology, 4. doi: 10.3389/fpsyg.2013.01017

Zaccarella, E., Papitto, G., \& Friederici, A. D. (2021). Language and action in Broca's area: Computational differentiation and cortical segregation. Brain and Cognition, 147, 105651. doi: 10.1016/j.bandc.2020 .105651

Zacks, J. M., \& Tversky, B. (2001). Event structure in perception and conception. Psychological Bulletin, 127(1), 3. doi: 10.1037/0033-2909.127.1.3 